EPJ Web of Conferences 95, 06002 (2015)

DOI: 10.1051/epjconf/20159506002

(C) Owned by the authors, published by EDP Sciences, 2015

\title{
Fifty years of research at CERN and elsewhere
}

\author{
D. Treille
}

${ }^{1}$ CERN, Geneva, Switzerland

\begin{abstract}
A brief overview of fifty years of particle physics at CERN and elsewhere is given.
\end{abstract}
\section{Synoptic view of fifty years of particle physics}

Table 1 shows, in chronological order, some of the most important steps and innovations in five different sectors: theoretical physics, experimental physics, machines, detectors and cosmology. I will focus on some topics and make short "guided tours" of their history. Much material will be drawn from [1].

Let me first point out the main trends in each sector, illustrated in the simpler Table 2.

Concerning physics, one can distinguish two contrasted periods. Until the early seventies, there was no or only a weak theoretical guidance. Once the quark model, the electroweak (EW) unification and Quantum Chromodynamics (QCD) were established, experimentation became testing, and up to now validating, the Standard Model (SM).

For detectors, it is clear that the 1968 revolution of G.Charpak and its MWPC opened a new era. One should also point out that nothing would have been possible without the exponential growth, in parallel, of the power of microelectronics and computing means.

It is also interesting to recall the main breakthroughs of astrophysics and cosmology. After the discovery of CMB in 1964, the paradigm of an expanding universe became dominant and this opened a new point of view on particle physics, namely that it performs, at a microscopic scale, some "archeology" of the very early instants of the universe, beyond the "curtain of invisibility". This is illustrated in Figure 1.

CERN scientific life can also be roughly split in two. A period of "apprenticeship", where CERN was learning how to create and run large collaborations and was building its technical strength. And, after the first big successes, like the discovery of Neutral Currents (NC), a period of maturity, which progressively led CERN to its present leadership at the high energy frontier of particle physics.

CERN lived several intense and euphoric moments: NC, the harvest of results from the SPS, the discovery of W and Z, LEP1 and LEP200, etc. Obviously LHC is now another one.

But CERN went also through some periods which, although intellectually stimulating, were quite depressing as seen from here: for instance, during the '1974 Revolution' which followed the discovery of the $\mathrm{J} / \Psi$ in the United States, one had the feeling that 'life was elsewhere'. Personally I also consider LEP200 as an unfinished symphony, as I shall illustrate later.

For long, CERN had a diversified physics program. Most physicists had several 'irons in the fire', analyzing an experiment, running another one, preparing a third. Currently, because of much longer time scales and in spite of the huge task, there is a risk of monoculture and boredom. Could young physicists share (unequally) their activity between the main line and a 'small' stimulating one? 
Table 1. Most important steps and innovations in fifty years of particle physics.

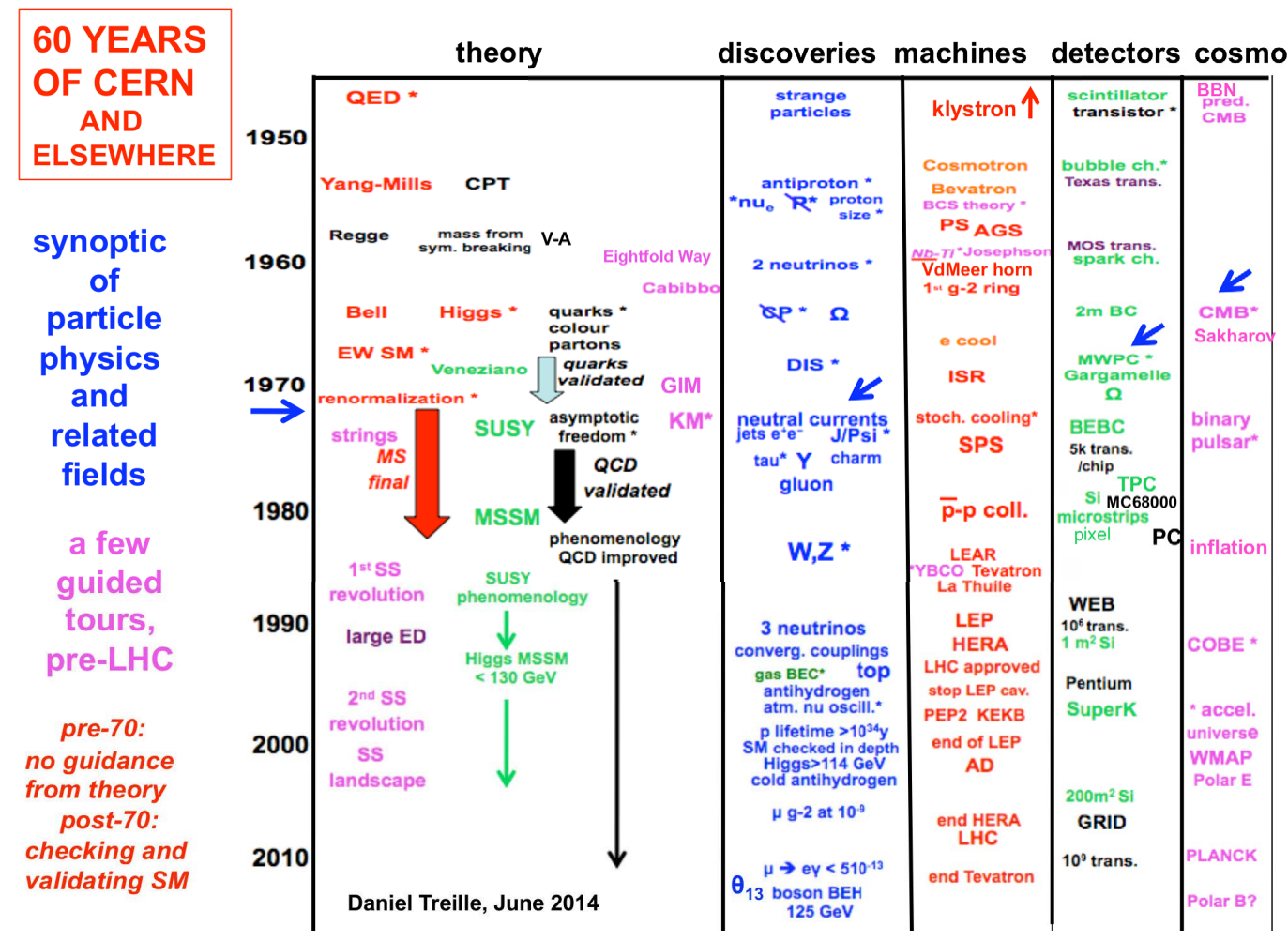

Table 2. A simplified view of the main trends.

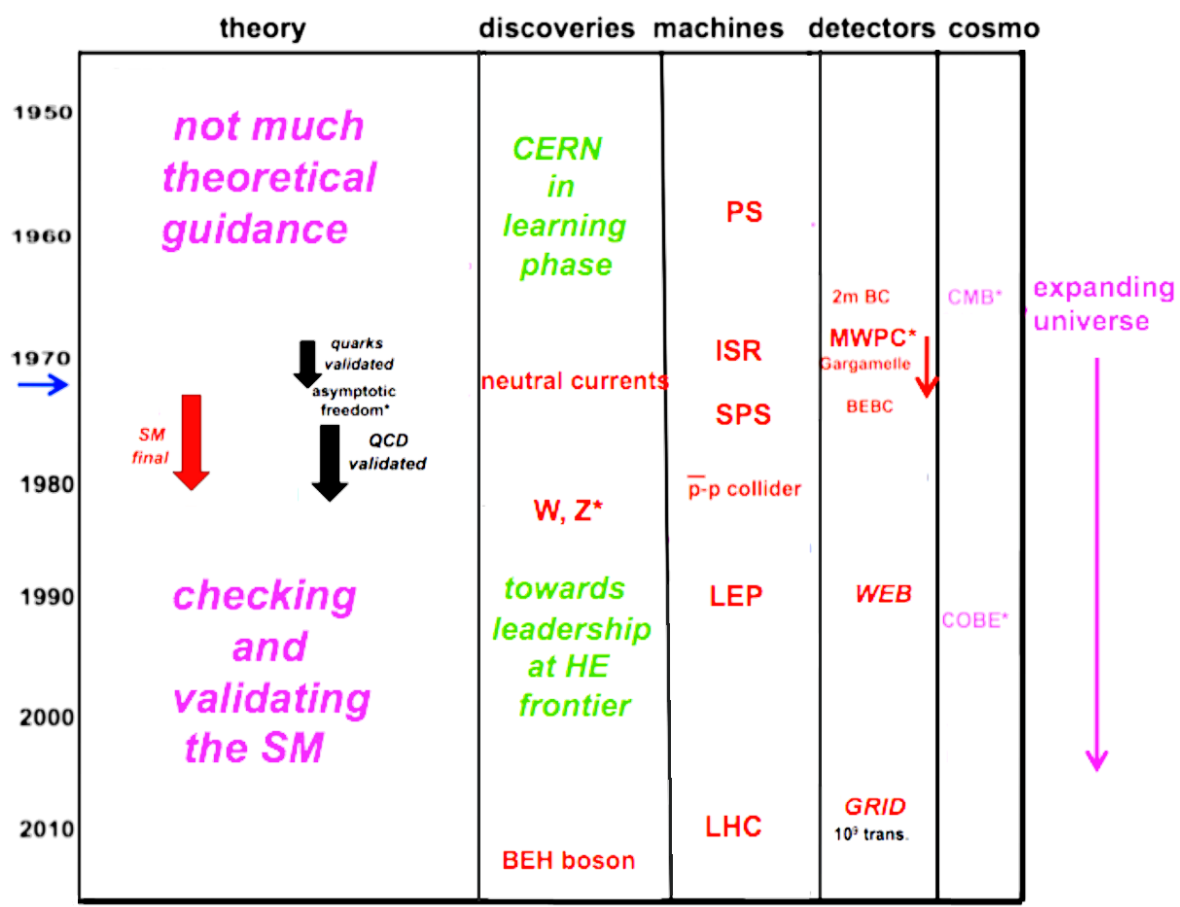




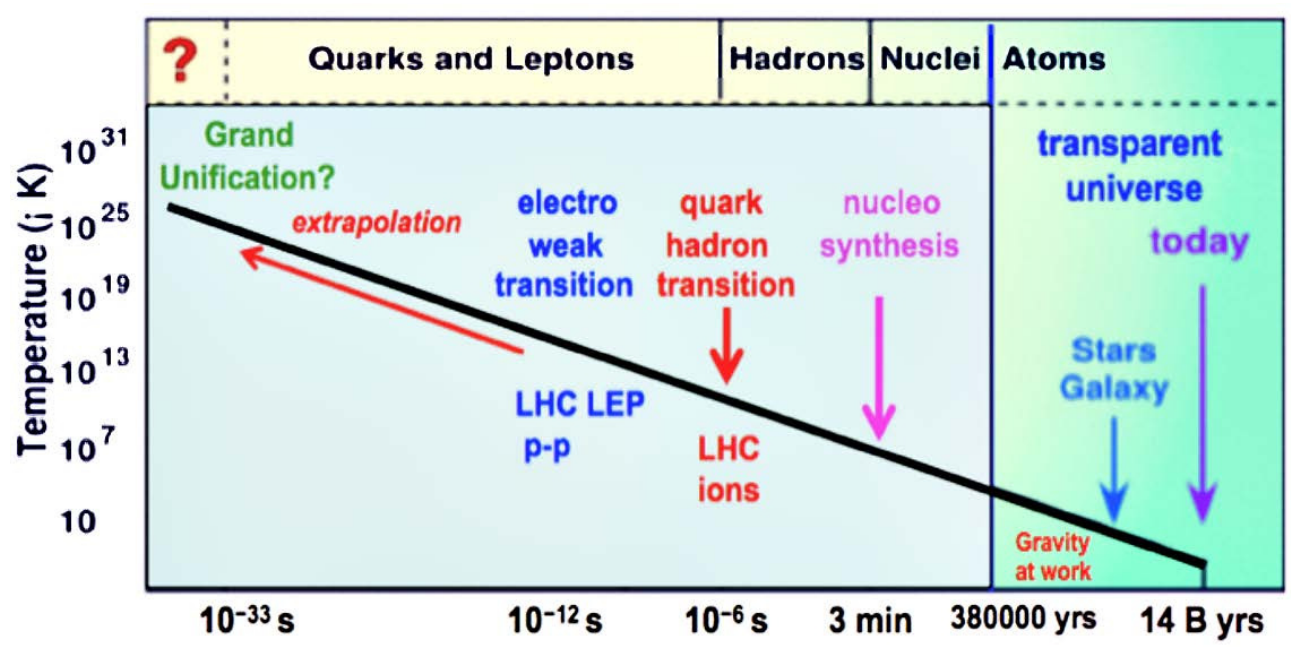

Figure 1. Particle physics located in the timeline of the Universe.

\section{A short history of the proton structure}

Figure 2 recalls the early discoveries from electron scattering: the finite size of the proton (1957, NP 1961) and the existence of point-like scatterers inside it (1968, NP 1990), leading to the early view of spin $1 / 2$ pointlike charged objects, called partons, later identified with quarks.

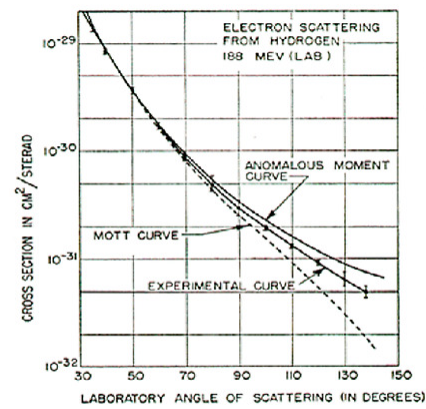

a

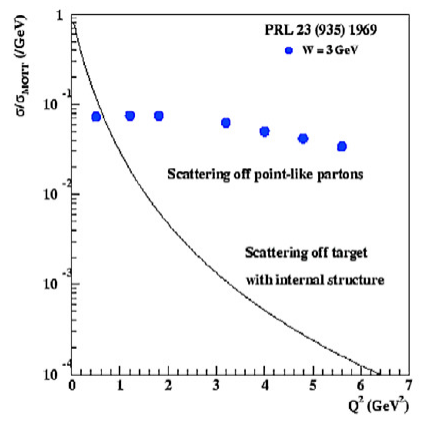

b

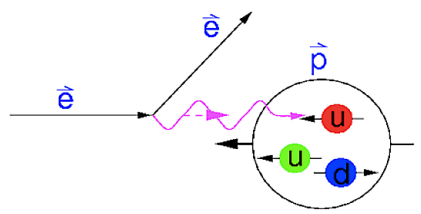

C

Figure 2. a: the proton is not a point; b, c: there are point-like scatterers inside it.

CERN was not involved in these early breakthroughs, but then it invested a lot in the detailed exploration of the nucleon inner structure, by using all possible probes.

Intense neutrino beams, thanks to S. van der Meer's horn, provided the weak probe (figure 3). They fed the giant bubble chambers, BEBC and Gargamelle, and large electronic detectors, as CDHS and CHARM, later CHORUS and NOMAD. The BC were the first to observe scaling violation.. 


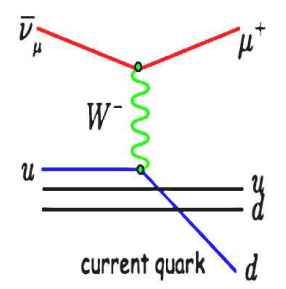

a

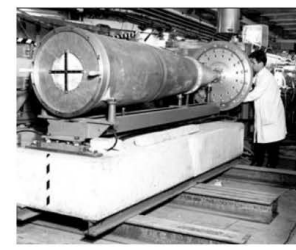

b

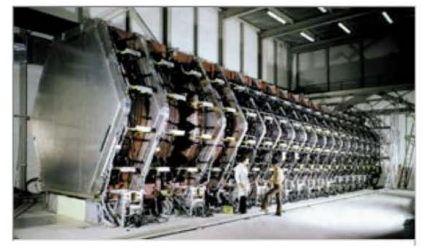

C

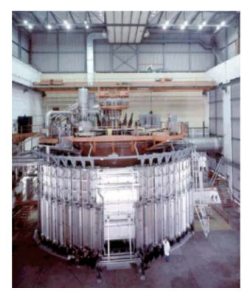

d

Figure 3. a: the weak probe; b: van der Meer horn; c: CDHS; d: BEBC.

The electromagnetic probe was provided by muon scattering. An intense high energy muon beam fed the EMC (European Muon Collaboration) and NA4. Besides a thorough study of nucleon structure functions, they discovered the EMC effect (the SF depend on the nucleus containing the nucleon) and showed that valence quarks contributed only to part of the spin of the nucleon.

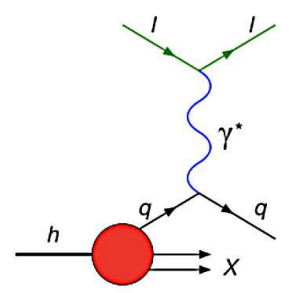

a

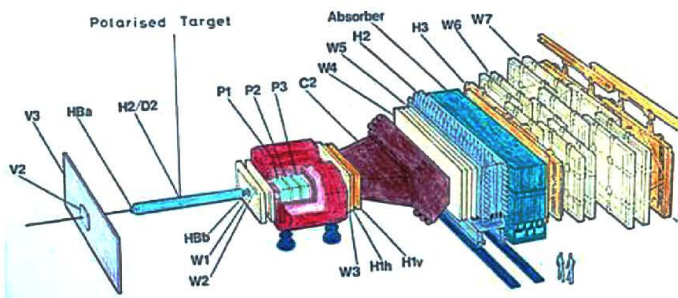

b

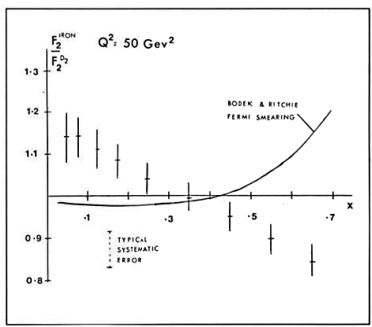

C

Figure 4. a: muon scattering; b: the EMC spectrometer; c: the EMC effect.

Another method of exploration of the nucleon was hadron or photon scattering, selecting in the final state "prompt" particles (photons, leptons) coming from the hard scatter. It allowed to study also the structure of the incident meson and discovered the K-factor, deviation from the expectation of the quark model (figure 5b). Fig 5c shows the QED Compton scattering on quarks.

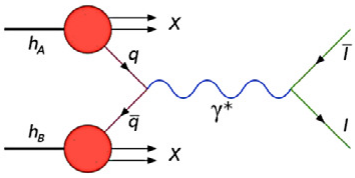

a

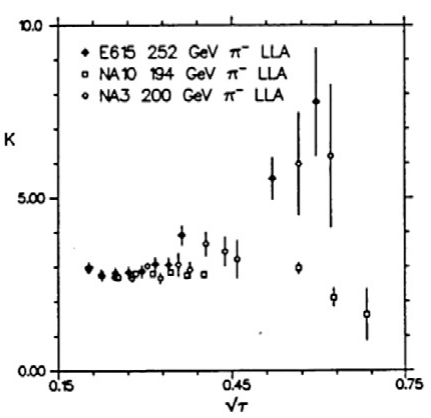

b

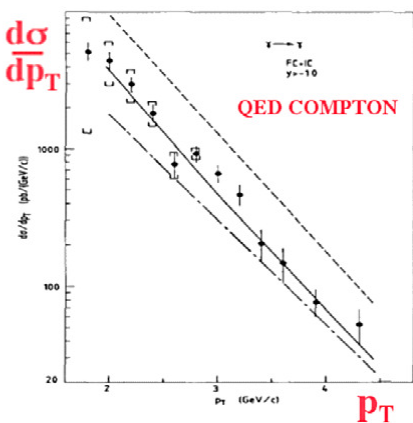

C

Figure 5. a: prompt leptons; b: $\mathrm{K}$ factor in pion-nucleon scattering; c: Compton scattering on quarks. 
More details on the vast SPS program can be found in Ref 1 .

Meanwhile it was realized that quarks have another quantum number called color (Greenberg 1964) and this gave birth to quantum chromodynamics (QCD), modern theory of strong interactions. The fact that gluons, mediating color between quarks, are also carrying the color charge and therefore self-interacting has dramatic effects: the confinement of quarks and gluons inside hadrons and the property of asymptotic freedom (1973, NP2004), telling that at short distance (high energy scale) the strong coupling between quarks decreases (figure $6 \mathrm{~b}$ ).

On the experimental side, the e-p Hera collider took over. Together, these programs led to the present view on the nucleon and provided a precise knowledge of its content in quarks, antiquarks and gluons, a key information for LHC physics

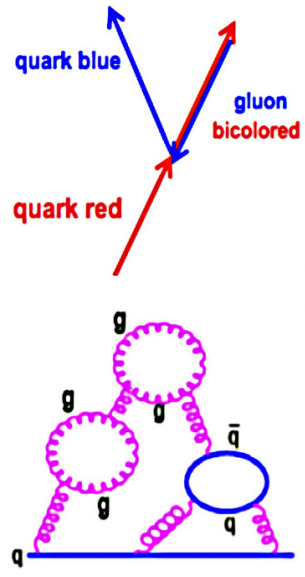

a

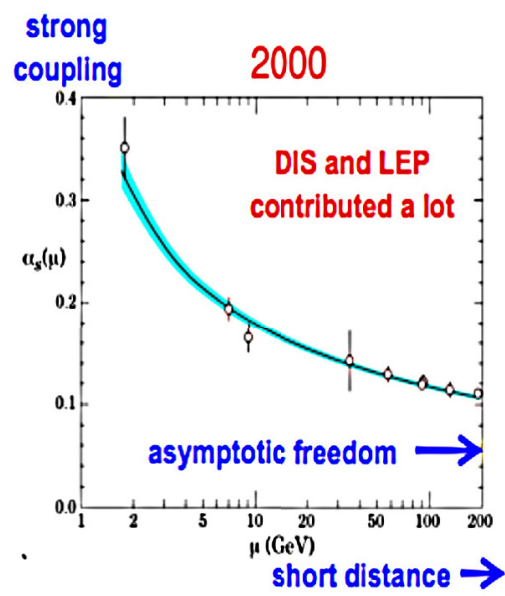

b
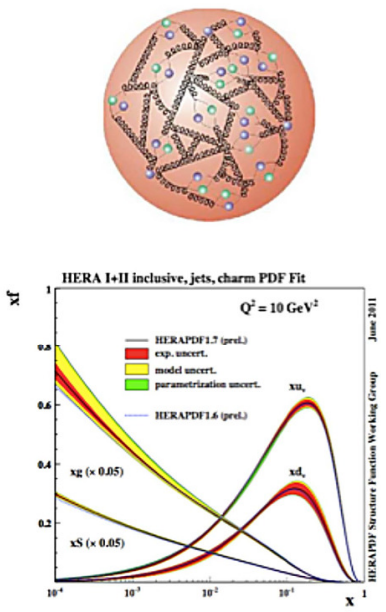

$\mathrm{C}$

Figure 6. a: the basics of QCD; b: asymptotic freedom; c: structure functions.

\section{The quark story}

In very brief, let us recall the steps which led from the discovery of a host of particles, in particular the strange particles, to the quark hypothesis (figure 7).

In 1953-1956 Nishijima and Gell-Mann invented the strangeness quantum number and gave the formula relating strangeness to electric charge, third component of isospin and baryon number.

Then, facing the abundance of particles and resonances, M.Gell-Mann and Y.Ne'eman understood in 1960 that, according to their quantum numbers, they could fit at the nodes of well defined patterns related to representations of the group SU(3). This group has 8 generators, and this classification was called the Eightfold Way. The tip of the baryon decuplet was still missing: the $ᄅ$ baryon was thus predicted in 1962 and discovered in Brookhaven in 1964.

Finally, in 1964, Gell-Mann (NP 1969), G.Zweig (and may be others, like A.Petermann) realized that all would be much simpler if one postulates the existence of three elementary building blocks with fractional electric charge, the quarks (or "aces" of Zweig), baryons being made of three quarks and mesons of a quark and an antiquark. Whether these were then considered as "real" objects is a matter of debate. However, after the discovery of point like scatterers in the nucleon, quarks were identified with partons and, once jets were observed, became "familiar" objects. The reduction à la Mendeleev of the particle bestiary had started. 


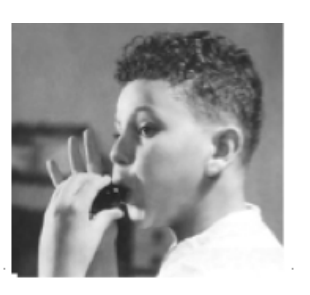

$Q=I_{3}+\frac{1}{2}(B+S)$

a

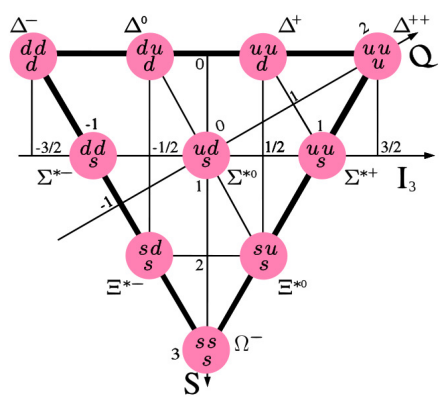

b

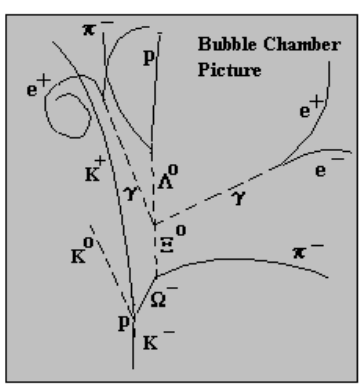

C

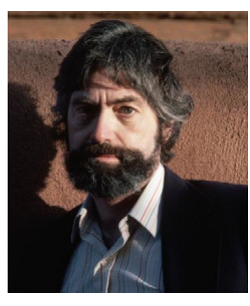

d

Figure 7. a: Gell-Mann and the Gell-Mann-Nishijima formula; b,c: Omega prediction and discovery ; d: G.Zweig

\section{The story of the three generations}

I will be also as brief as possible. With strangeness and the muon, it was clear that more than the first generation was existing. To explain the features of strangeness production, Cabibbo introduced in 1963 his mixing angle (figure $8 \mathrm{~b}$ ).

The existence of a $4^{\text {th }}$ quark, charm, was suspected (Bjorken-Glashow, 1964) and was put on firm grounds by GIM (Glashow, Iliopoulos, Maiani, 1970), realizing that its existence and role would be the best explanation of a mystery: the non observation (at that time) of the decay of $\mathrm{K}^{0}$ in two muons.

In 1974 charm was discovered at SLAC and Brookhaven (NP 1976), but as charmonium, bound state of charm and anticharm. Although reminiscent of the ᄊ vector meson, the very narrow width of the J/Psi was a surprise for many. It started the industry of "onium" spectroscopy. Charmed particles appeared in 1976. The second generation was there.

In 1975 came the tau lepton (M.Perl, NP1995), telling that there was more than this second generation. But, before that, Kobayashi-Maskawa (1973, NP 2008) postulated the existence of a third one, with very good reasons to do so. Beauty was found in 1977. We will discuss the top later. LEP showed in 1990 that there are indeed 3 neutrino species (active and lighter than $M_{Z} / 2$ ).

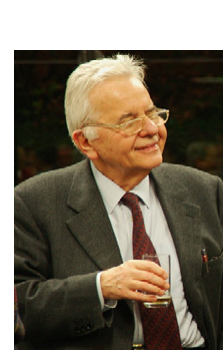

a

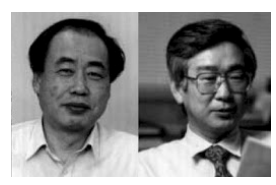

e

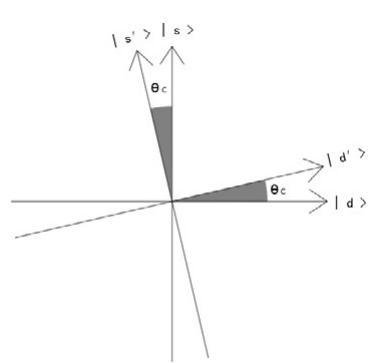

b

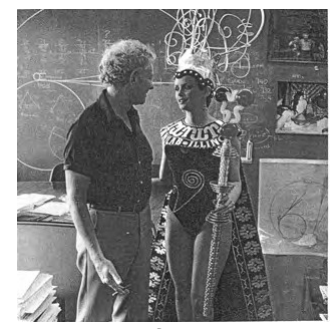

f
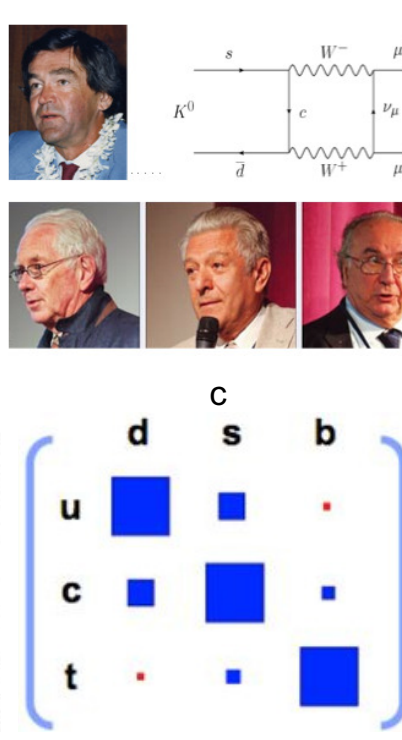

g

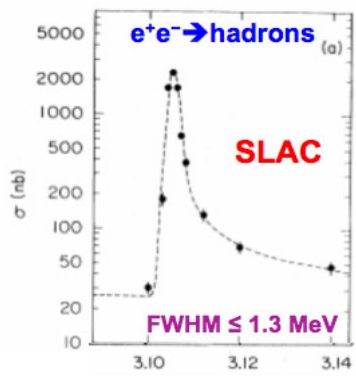

d

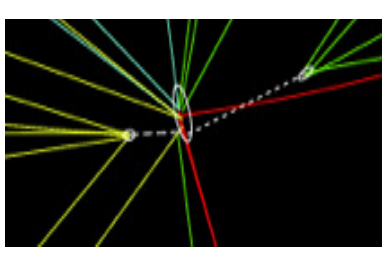

$\mathrm{h}$

Figure 8. a: N.Cabibbo; b: Cabibbo angle; c: Bjorken and GIM; d: the Psi from SPEAR; e: Kobayashi and Maskawa; f: Lederman discovering beauty; g: the CKM matrix; h: short lived particles. 
Flavour particle physics entered then its modern era, with the parameterization and progressive filling of the CKM matrix, telling how quarks "communicate" with each other. The big bonus of a third generation is that the matrix contains a phase, which is the key of the possibility of CP violation, and therefore, according to Sakharov, a prerequisite to our own existence... Except maybe for righthanded neutrinos, there is presently no need of more elementary constituents.

Charm and beauty particles live about a picosecond. This opened the field of heavy flavour "tagging" with precise detectors close to the interaction point. CERN played there a major role.

\section{The SM story}

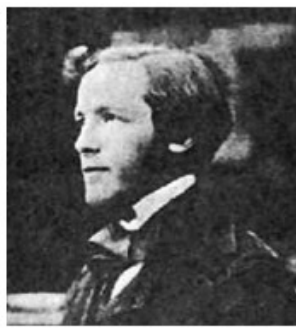

$$
\begin{aligned}
d F & =0 \\
\star d \star F & =J
\end{aligned}
$$

a

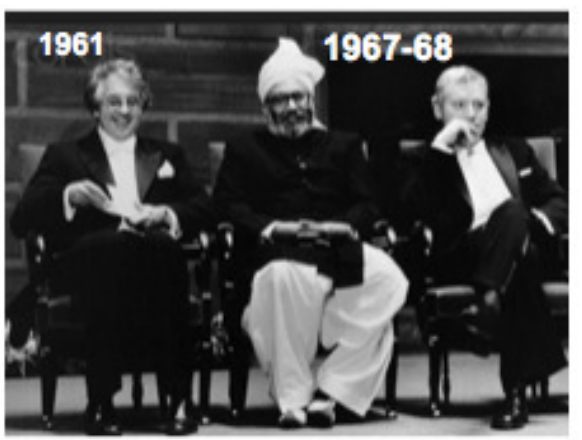

d

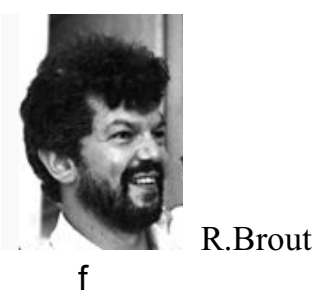

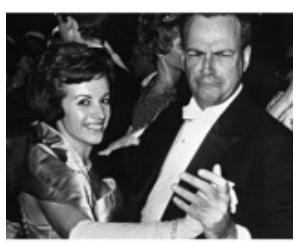

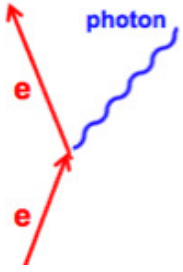

b
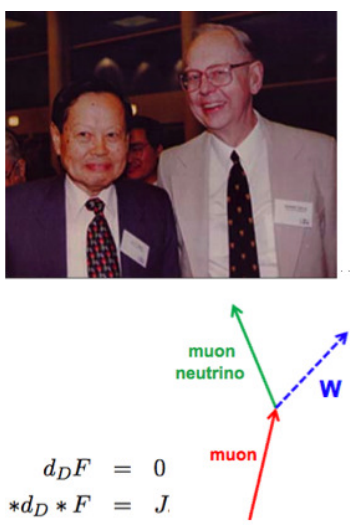

C

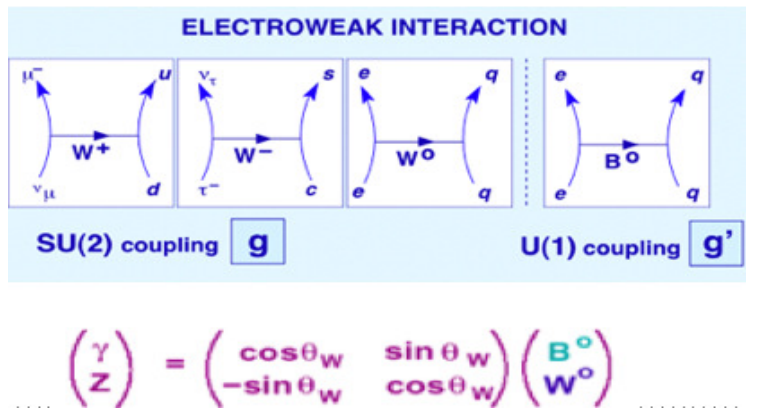

e

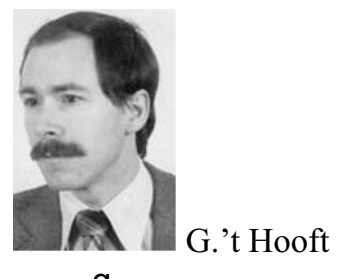

g

Figure 9. a: Maxwell and his equations; b: Feynman and QED; c: Yang-Mills and covariant equation; d: Nobel 1964: Glasow, Salam, Weinberg; e: the EW SM algebra and the weak angle; f: R.Brout, co-inventor of the BEH boson, deceased in 2011; g: G.'t Hooft. 
Figure 9 leads us from Maxwell equation (1861) (here in differential form) to Quantum Electrodynamics (QED) the first complete quantum theory (Feynman et al, 1949, NP 1965) accounting for charged fermion -- photon interaction. The key point is the requirement of local invariance of the theory, implying the existence of a vector force carrier (here the photon) to transmit the interaction.

Then Yang-Mills (1954), with the same idea of local invariance, extended the theory to the case where the carrier of the force is also carrying the corresponding charge, and thus self interacts. These are local non-abelian gauge theories, as for instance the EW theory and QCD.

Building on that, the SM of electroweak interaction was conceived in two steps. In 1961 the correct algebra of the EW theory was given by Glashow. But it still worked only for massless objects.

In a second step (1967-68), the idea of spontaneous breaking of symmetry, the Brout-EnglertHiggs (BEH) mechanism (1964, NP 2013) was incorporated by Salam and Weinberg (NP1979 with Glashow), predicting massive $\mathrm{W}$ and $\mathrm{Z}$ bosons. The renormalizability of the theory was proven in 1971 by G.’t Hooft, NP 1999 with M.Veltman.

The mission to experiment was clear: find the $\mathrm{Z}, \mathrm{W}$ and the BEH boson. In this respect, CERN did very well.

\section{The mutations of the detector methods}

One should remember that the ancestors of modern detectors are not so far away. While the Wilson chamber was born in 1911 and the Geiger-Muller counter in 1928, one had to wait until the years 1947-50 to see the first scintillators, the Bubble Chamber (BC) was invented in 1953 (Glaser, NP 1960), the MultiWire Proportional Chamber (MWPC) in 1968.

The first two decades of CERN life were the reign of bubble chambers. While the first was partly apprenticeship, the second decade saw the successes of the liquid hydrogen (LH) and heavy liquid (HL) large chambers. By the end of the life of this technique, rapid cycling BCs were successfully used for charm physics. The methods of analysis of their pictures were evolving in parallel and deserve a paper by themselves (quoted in [1]).

Triggered detectors then entered the game. From 1949 to 1959 spark chambers (SC) were developed as tracking devices. The first massive use of SCs was at BNL in 1962, when evidence for the second neutrinowas found. The various readout methods changed from film to filmless ones: acoustic, vidicon, wires. The OMEGA optical spectrometer was operated in 1972. In 1964 appeared the first online computers.

1968 saw the 'revolution' of G. Charpak (NP 1992), inventor of the MultiWire Proportional Chamber (MWPC), which then led to the drift chamber, the multistep chambers, a series culminating with the time projection chamber. The first large-scale use of MWPC occurred in 1971: the CERNHeidelberg experiment, by J. Steinberger et al., at the PS on CP violation and, at the ISR, the Split Field Magnet, suggested by J. Steinberger and used by G. Charpak, A. Minten, P.G. Innocenti et al. (unfortunately the detector was blind around $90^{\circ}$ ).

Cherenkov detectors, from threshold devices and focusing ones (DISC) to the Ring Imaging technique (RICH), have played a key role in particle identification. The RICH of T. Ypsilantis and J. Seguinot, after being used during the LEP era (DELPHI, SLD, heavy ions), seems to have many descendants, an example being in LHCb.

Concerning calorimetry, important breakthroughs were the understanding and exploitation of the compensation mechanism and the move towards pointing geometries, from the 'Spaghetti' to the ATLAS 'Accordeon'. Liquid argon and scintillating crystals are dominating the field at colliders.

Hybrid systems, from BEBC to the European Hybrid Spectrometer, have played an important role, in particular for charm physics.

Currently the ongoing revolution is the rise of silicon detectors. While the goal was flavour tag in the LEP era, one is now using them as main tracker. This represents a big step, from the square metre typical of a LEP microvertex to hundreds of square meters, as for the CMS tracker at the LHC. 
Clearly this progress was made possible by various breakthroughs occurring in microelectronics, in particular the advent of submicron processes, intrinsically radiation hard, a very welcome feature for LHC experiments. Silicon detectors are also considered for calorimetry.

In all these mutations the crucial role of detector R\&D (in particular the strong DRD program undertaken to learn how to exploit the full luminosity of LHC) is obvious. It is clear as well that, if we want to progress further, some R\&D has to continue. Whatever the physics goal may be (SuperLHC, ILC, CLIC, future fixed targets), some of the requirements are similar. For an optimal tracking, one needs real 3D information obtained from pixels, micro or macro, rather than from stereo microstrips, vectorial information (position and direction), thinner, faster, harder and cheaper detectors. As for calorimetry, one is still far from a completely satisfactory design regarding its granularity and the integration of the electromagnetic and hadronic parts.

Figure 10 below give an idea of the evolution with time of the various classes of detectors. What is plotted in 10-a and 10-b is simply the number of entries in the search engine Spires whose title refers to a given technique. The fall of the curves does not imply that the technique is no longer used, but means that it no longer deserves a publication by itself.

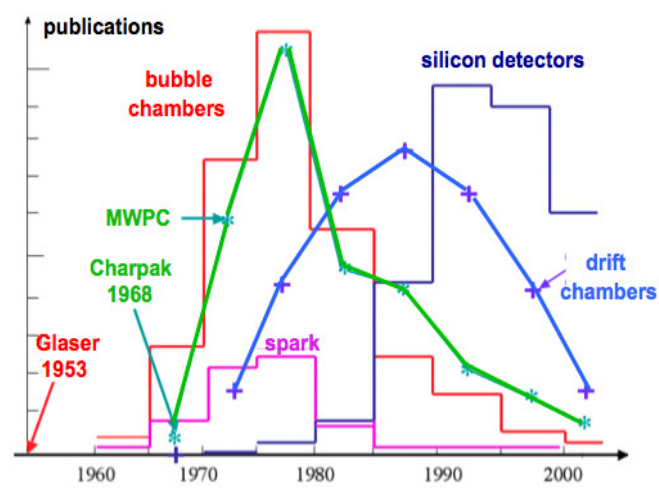

a

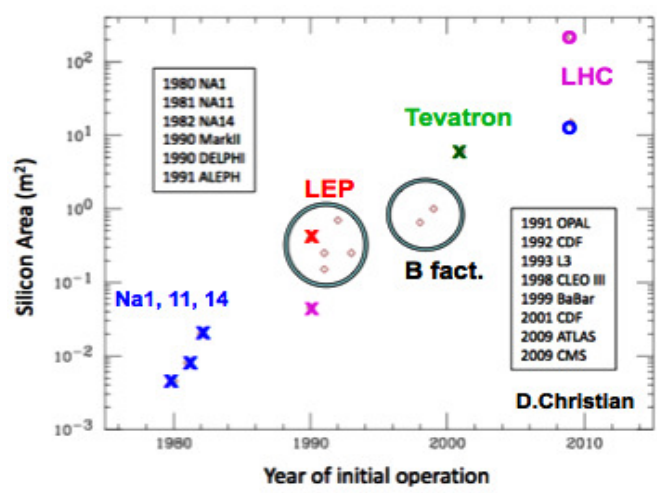

C

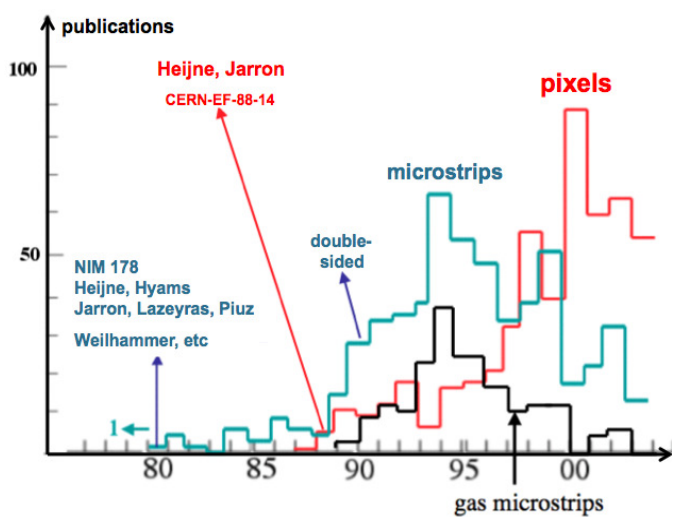

b

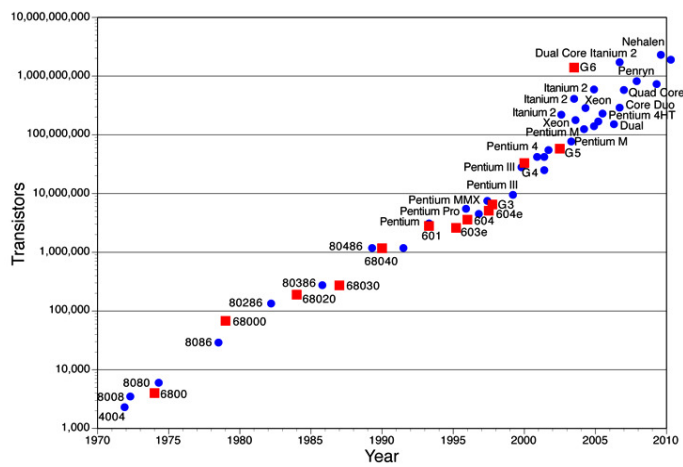

d

Figure 10. a: evolution with time of the various types of detectors; b: more details on silicon detectors; c: area of Si detectors along the years; $\mathrm{d}$ : the progress of microelectronics. 
Heavy flavour physics was a major activity of CERN, in all its programs. We will mention later B physics at LEP. The B factories took over, and the domain is presently dominated by LHC, in particular LHCb. However one should underline the heavy flavour physics, mostly of charm, performed at the SPS, because of the intense activity and creativity in the field of Si detectors, which paved the way to the future enterprises. See [1] for more information.

\section{The Neutral Currents (NC)}

I borrowed much from several excellent chronicles about Neutral Currents [2].

From the $1.2 \mathrm{~m}$ hydrogen chamber, there was an upper limit on the ratio of elastic NC to Charged Current (CC) events $(<0.03)$, published in 1964 . This was simply a mistake uncovered by M. Paty in 1965.

After the Siena 1963 conference, A. Lagarrigue, A. Rousset, P. Musset worked out a proposal of a neutrino detector aiming at increasing the event rate by an order of magnitude. L. Leprince Ringuet called it Gargamelle. The team was composed of physicists from X-Orsay and of former members of the NPA $1 \mathrm{~m}$ BC. It included seven European laboratories and one guest laboratory.

In 1971-72 a viable theory of weak interactions existed and the question was clearly posed: are there NCs, yes or no? The competitors in the field were Gargamelle and the HPWF counter experiment at NAL.

Even in November 1968, at a Gargamelle meeting in Milan, the word NC was not pronounced. This problem had low priority and Deep Inelastic Scattering (DIS) was more exciting then.

But in 1971 everything was ready. A careful classification of event types was devised. NC events looked like neutron star events without a muon. The goal was to separate neutrino-induced from neutron-induced stars.

In December 1972 one electron event was found. The search for hadronic NC candidates went on. Candidates were observed, with a flat spatial distribution, and caused much excitement. However, counter arguments were put forward and one realized that the only way to conclude was to prove that the number of neutron-induced events was small. Monte Carlo simulation then showed its strength: a neutron background program with no free parameter was used, complemented by other approaches. It allowed the conclusion that the NC sample was not dominated by neutron stars (J. Fry and D. Haidt, CERN Report 75-01, fig 11 g). At the Bonn 1973 conference, C.N. Yang announced the discovery of Neutral Currents.

However, by that time the HPWF signal had disappeared. This threw dismay and distrust on the whole matter, and one more year of work was needed to conclude. Finally, at the London 1974 conference, evidence for NC was confirmed by Gargamelle and HPWF.

This major discovery established the similarity in structure of the weak and electromagnetic interactions. The term 'electroweak' came into being. This was the experimental beginning of the SM. From the ratio of $\mathrm{NC}$ over CC one could estimate the Weinberg angle. By 1982-1983 $\sin ^{2} \mathrm{r}_{\mathrm{W}}$ value was narrowed down to around 0.23 , predicting $\mathrm{m}_{\mathrm{w}} \sim 80 \mathrm{GeV}$ and $\mathrm{m}_{\mathrm{z}} \sim 90 \mathrm{GeV}$ to within a few $\mathrm{GeV}$.

The NC adventure is probably a "missed" Nobel Prize because of the premature death of A.Lagarrigue

One must also realize that the success of Gargamelle was only possible because of the long experience on BC built at CERN and its collaborating laboratories since the early days. The saga of $\mathrm{BC}$ at CERN deserves much recognition. The young reader can find more in [1] (and references therein). 


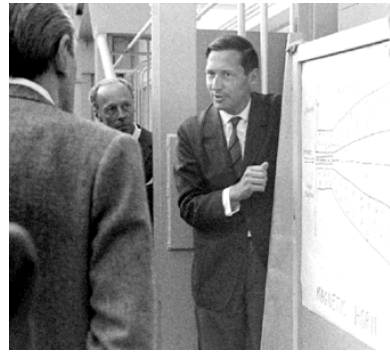

a

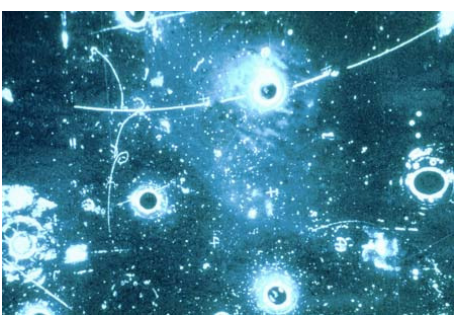

d

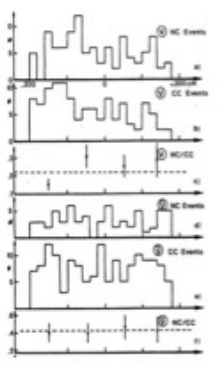

g
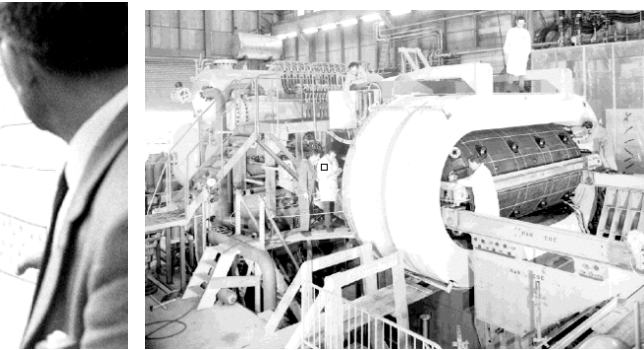

b

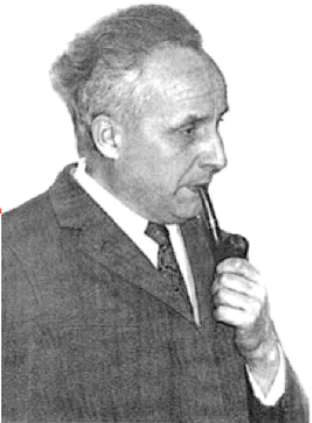

C

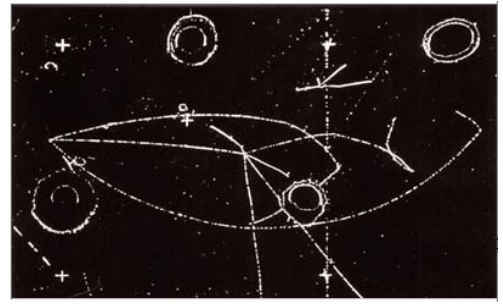

e

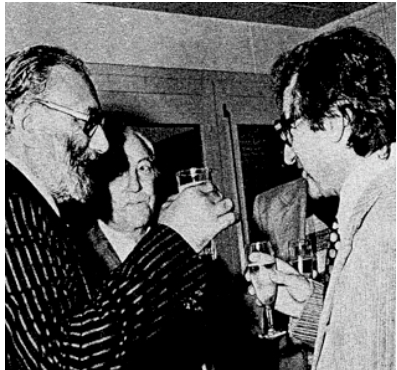

h

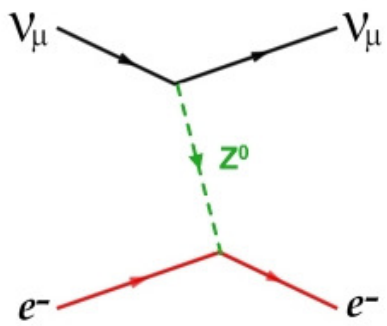

f

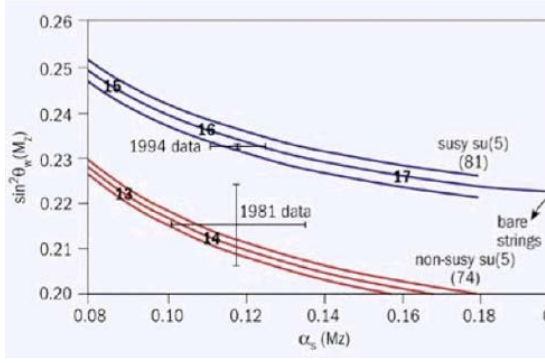

i

Figure 11. a: S. van der Meer; b: Gargamelle; c: A.Lagarrigue; d: an electron event; e: a hadronic event; f: $Z^{0}$ exchange; g: neutrinos versus neutrons along the chamber depth; h: A.Salam and P.Musset; i: "ad augusta per angusta".

Besides Neutral Currents the large bubble chamber brought other most interesting results.

By comparing the structure functions of the nucleon derived from neutrino and muon scattering, Gargamelle proved that quarks have indeed a fractional electric charge. Gargamelle demonstrated that the neutrino-nucleon cross-section rises linearly with energy. Finally, by combining their results, BEBC and Gargamelle got the first evidence for scaling violation, namely the proof that the nucleon structure functions actually evolve with the resolution power of the observation.

An amusing fact is illustrated by Figure 11 i. In 1981, the value found for the Weinberg angle was in agreement with non-supersymmetric Grand Unification. This predicted a rather low GU scale and a proton lifetime of $10^{30}$ years or so. This prompted programs aimed at detecting proton decay, like Kamiokande. They did not find proton decay because this value of the Weinberg angle was actually incorrect. But they later observed the first supernova neutrinos and discovered the non-zero neutrino mass via neutrino oscillations! 


\section{The ISR}

The Intersecting Storage Rings (ISR) was the first hadron collider ever built. M. Jacob sees:

- "A brilliant start": 1971-74.

An early discovery at the ISR was the rising total proton-proton cross-section in 1971 . The data led to conclude that, while the proton size increases with energy, its profile stays the same.

Another major finding was the discovery of the large PT phenomena at the ISR in 1973: it demonstrated that partons were behaving as pointlike objects for the strong interaction as well as for the electromagnetic one.

The study of Diffractive Dissociation told how energy hadronizes, independently of the nature and energy of the colliding particles.

- "A somewhat difficult period": 1975-77.

The reason is that several big discoveries $(\mathrm{J} / \psi$, charm, beauty) within reach of the ISR were made in the US, in part because the ISR detectors were not adequate.

- "A very active and interesting programme": 1978-83.

Among other topics one can mention the studies of lepton pair production: $\mathrm{J} / \psi, \mathrm{Y}$, dilepton masses up to $20 \mathrm{GeV}$, properties of the Drell-Yan process. However, charm and beauty semileptonic decays were ignored in these data analyses. Concerning the production of heavy quark bound states, like $\mathrm{pp} \rightarrow \chi_{\mathrm{c}}$, the results obtained at that time are still competitive.

The first clear mass peaks of charmed hadron production in hadronic interactions were observed in 1979, following several earlier indications from the measurements of single electron and lepton pairs. However, the charm cross-section determination turned out to be troublesome. The production of a beauty baryon was announced but is a controversial matter.

The production of direct photons was studied, from their discovery in 1979 to the end of the ISR. These measurements played an important role in testing QCD. Gluon Compton scattering was found to be dominant, the sub-leading process being quark-antiquark annihilation. This fact allowed the determination of the gluon distribution inside the proton. The study of the two-photon final states was also performed. A comparison of the yields with antiproton-proton collisions would have been interesting, but unfortunately the luminosity was insufficient.

Concerning the evidence for jets in hadronic collisions, it started with a rather confusing period, both in fixed-target experiments and at the ISR. By 1982 the AFS and R110 experiments at the ISR had an appropriate acceptance to see jets. But the results of the proton-antiproton collider came at about the same time, and these 'stole the limelight', although different x-regions were probed in the two sets of measurements.

Whatever be the area of the physics programme, the machine worked magnificently: the ISR were a most efficient R\&D laboratory for accelerator physics. In particular, it allowed the testing of the idea of stochastic cooling, key of later successes.

By the end of the ISR in 1983, M. Jacob, quoting Mark Antony, in Shakespeare's Julius Caesar, said: "I come to bury Caesar not to praise him", while V. Weisskopf argued that it does not matter where discoveries are made.

Nevertheless, the morale at CERN was low. In one of the pictures, V.Weisskopf seems to exhort us to turn to superior instances. That is what was done in a quite oecumenical way. 


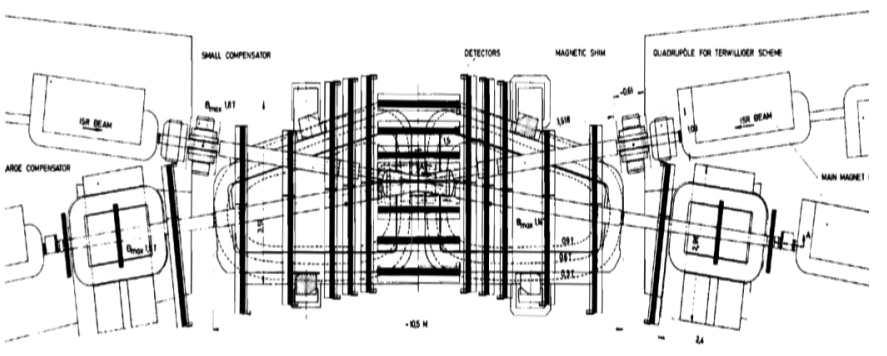

a

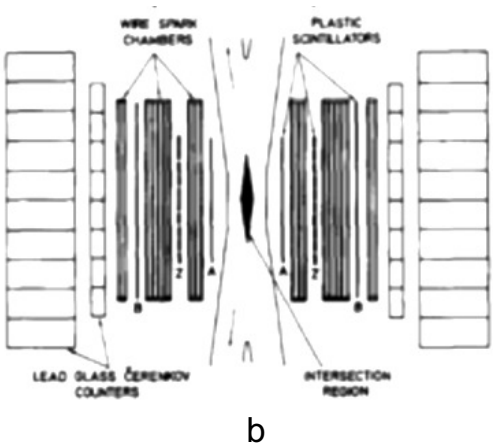

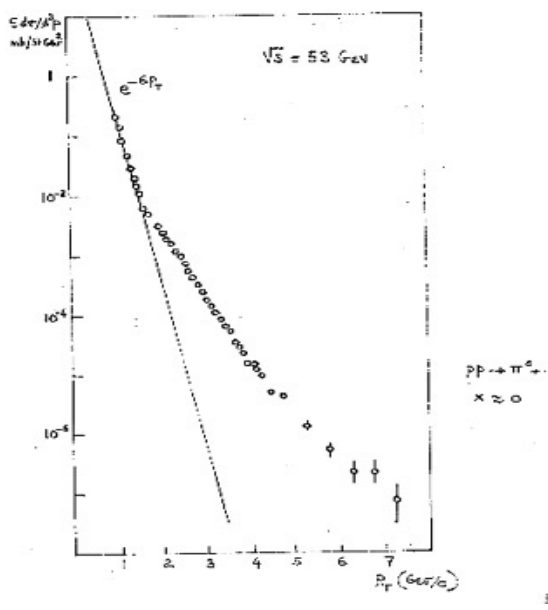

C

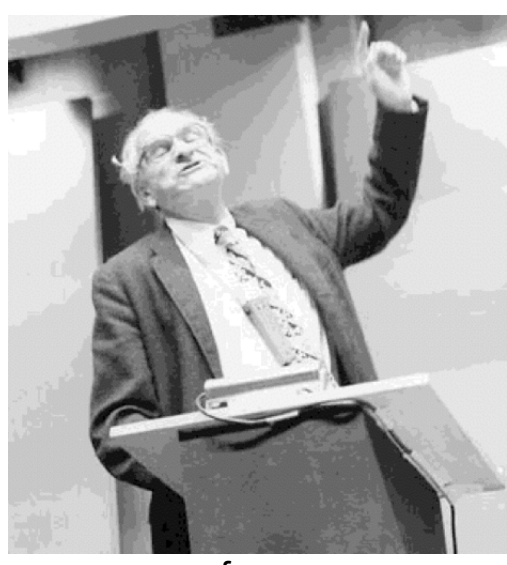

f

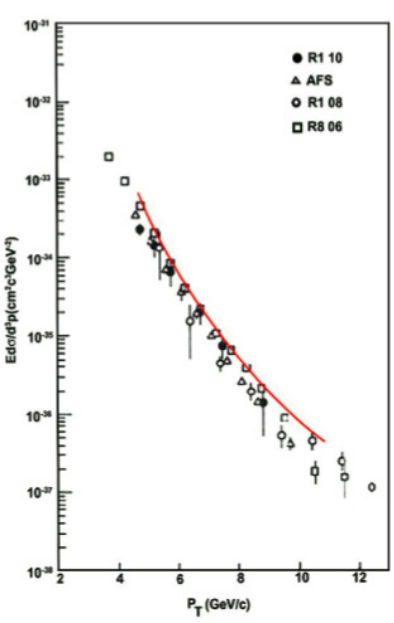

d

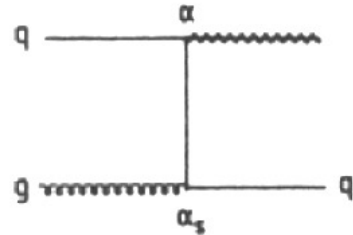

(a)

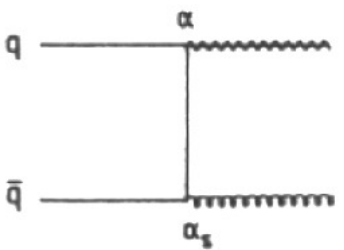

(b)

e

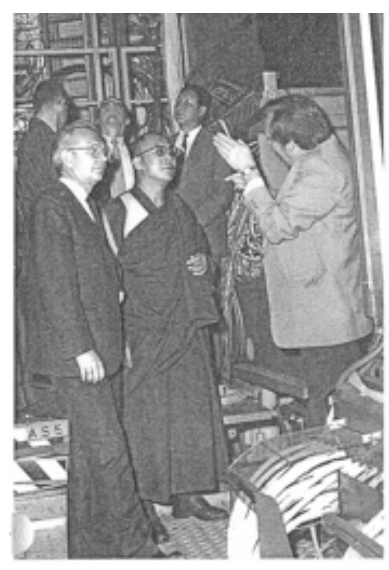

g

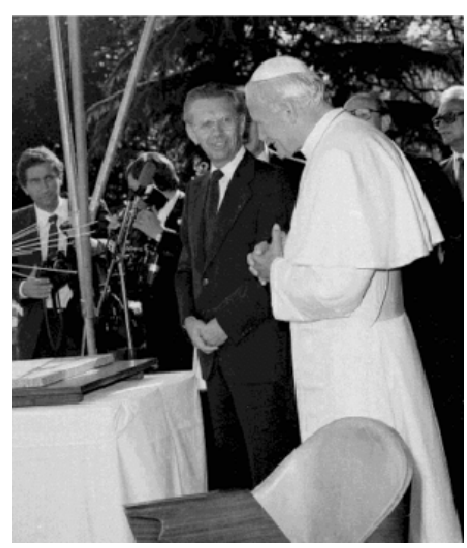

h

Figure 12. a: the split-field magnet; $b$ : the CCR spectrometer; $c$ : the high $\mathrm{p}_{\mathrm{t}}$ signal at the ISR; $d, e$ : direct photons and dominant diagrams; f: Weisskopf exhortation; g, h: prestigious visitors. 


\section{The proton-antiproton collider}

Actually the real cure came from the success of the proton-antiproton collider.

A look at the synoptic tables shows how fast were the decision-making and the realization of this program. The collider was a great machine, largely due to the use of stochastic cooling. It fed two outstanding and quite innovative detectors. For the first time, concepts like hermeticity of a detector, redundancy of the procedures, etc, were fully taken into account. The figures below show the UA1 and UA2 detectors and UA1 powerful tracker.

As we all know, "la physique était au rendez-vous". Besides the most celebrated discovery of the W and Z bosons (1983, NP 1984 to C.Rubbia and S. van der Meer), close to the predicted masses, the collider brought a clear picture of hard hadronic scattering.

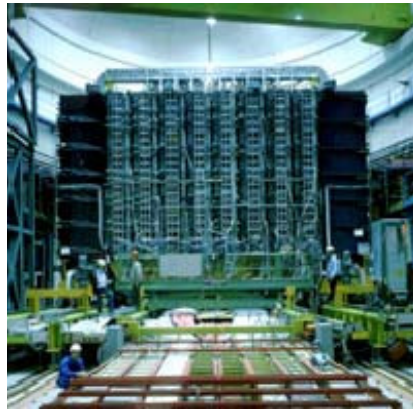

a

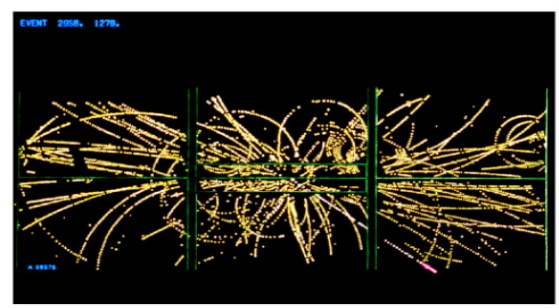

d

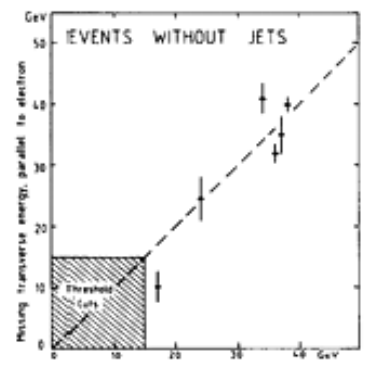

g

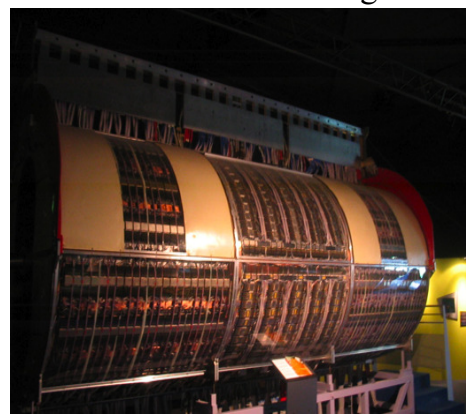

b

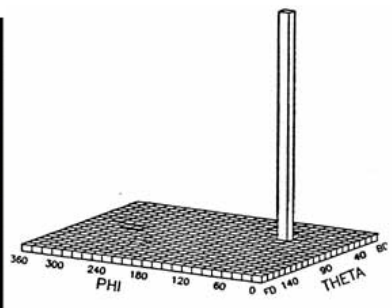

e

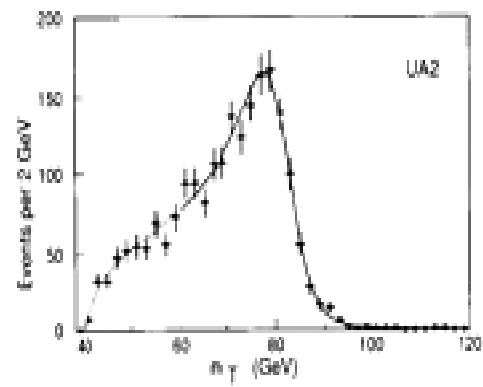

$\mathrm{h}$

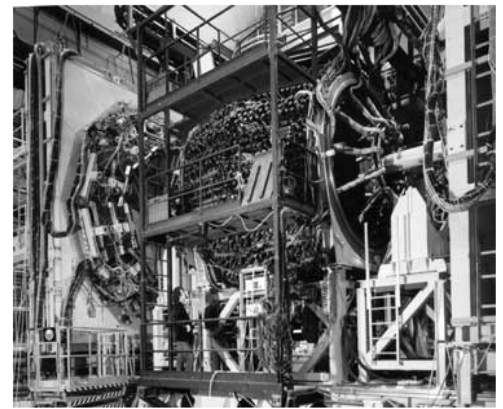

C

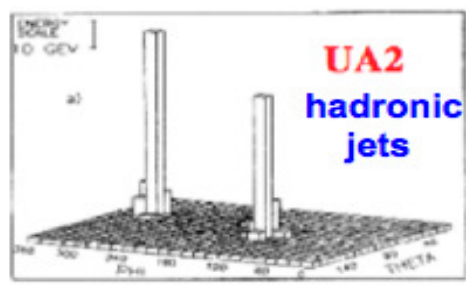

f

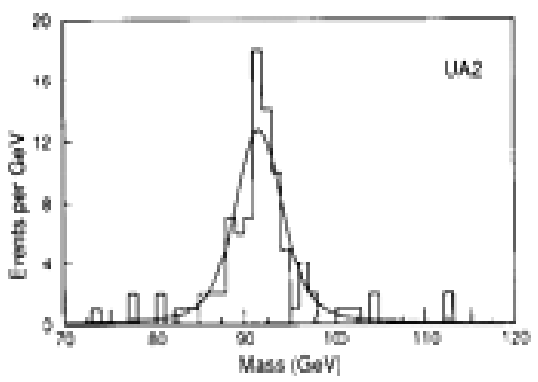

i

Figure 13. a: UA1; b: its tracker; c: UA2; d, e: a W; f: 2-jet event; g, h: W first evidence and final measurement; $i$ : the $Z$ final mass spectrum in UA2.

In 1981 the fixed-target NA5 experiment, with full azimuthal calorimetric coverage, did not see any jet structure. The situation in hadronic scattering was thus very different from the one of $\mathrm{e}^{+} \mathrm{e}^{-}$ scattering in which a clear two-jet structure had been observed since 1975. Even after the first run of the collider the results were not decisive. However, in 1982, first UA2, then UA1 obtained clear, 
uncontroversial evidence for jets. UA2, for instance, had a full azimuthal and a $40^{\circ}$ to $140^{\circ}$ polar angle coverage.

Many results then followed, among them:

- the determination of the angular distribution of parton-parton scattering,

- the determination of the proton structure functions, showing the role of gluons at small $\mathrm{x}$,

- the production of direct photons, found to agree with NLO QCD,

- the measurement of the total pt of two-jet systems, agreeing also with QCD,

- the studies of multijet final states, of the $\mathrm{W}$ transverse momentum, of heavy flavour production (beauty), all behaving as expected.

The collider thus proved the physical reality of partons inside the proton and opened the door to quantitative tests of QCD.

Much progress has been obtained since then, in particular by the Tevatron proton-antiproton and HERA electron-proton colliders. What remained to be done, concerning QCD, in order to guarantee a proper search program at the LHC, is impressive as well.

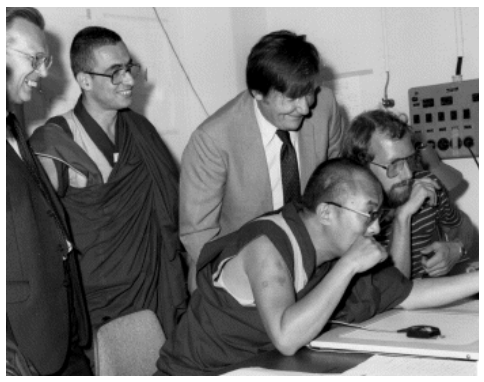

a

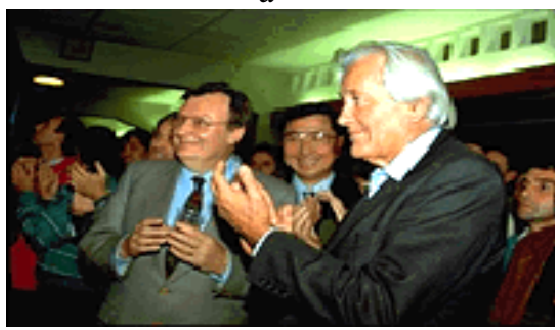

C

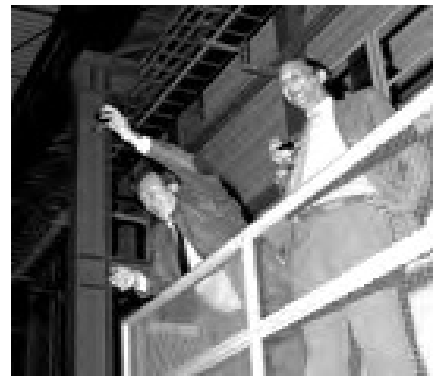

b

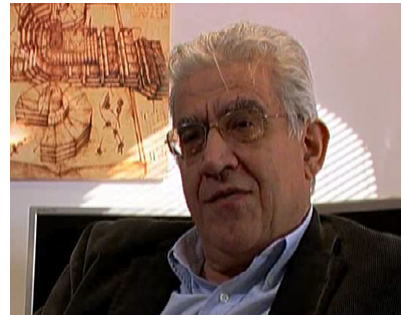

d

Figure 14. Figures from the collider. a: at the Megatek; b: C.Rubbia and S.van der Meer; c: C.Rubbia, S.Ting, G.Charpak; d: S.Cittolin.

\section{Accurate tests of gauge theories: g-ology}

The $g-2$ programs have extended over 40 years and still go on. As John Adams once said: " $g-2$ is not an experiment: it is a way of life". Indeed some of the initiators are still currently involved. From my point of view it represents one of the most beautiful achievements in particle physics, experimentally and theoretically.

The $\mathrm{g}$ factor is the constant that determines how much magnetic moment $\mu$ results from a given amount of charge, mass and spin $\mathrm{s}, \mu=\mathrm{g}$ e $/ 2 \mathrm{~m} \mathrm{~s}$. For the electron or muon, the Dirac equation gives $\mathrm{g}=2$. Actually a slight difference arises because of radiative phenomena (Fig. 17) and the $\mathrm{g}-2$ quantity turns out to be an amazing testing ground of theory, QED and EW, since all interactions contribute to it. The present measurements of $(g-2) / 2$ are

- electron: $(1159.65218076 \pm 0.00000027) \cdot 10^{6}$, measured to $2 \cdot 10^{10}$

- muon: $(11659209 \pm 6) \cdot 10^{10}$, measured 'only' to $5 \cdot 10^{7}$, but with the potentially interesting effects boosted by the mass squared factor (40 000).

The confrontation between experiment and theory has been a "tennis game with well-matched 
players on either side of the net", actually a 40-year long experimental program matching a 40-year long program of more and more refined calculations. At first order: $\alpha / 2 \pi \sim 0.00116$, giving $\mathrm{g}=2.00232$. There are 7 two-loop diagrams, 72 three-loop diagrams computed in 1995 by T. Kinoshita at Cornell (at better than one part per million), 891 four-loop diagrams, for which numerical computations have been under way since early 1980, and 12672 five-loop ones. The role of hadronic light-by-light scattering is important and actually leads to the present residual ambiguity in the interpretation.

The value of $g-2$ is obtained by measuring the beat between the rotation of muons around a ring and the rotation of their spin (figure 16). The movement of the spin is described by the BargmannMichel-Telegdi equation.

In 1958 a first experiment was done at the SC. The actors are shown in fig 15a.

In 1963 came the idea of a new experiment at the PS with $1.2 \mathrm{GeV}$ muons involving E. Picasso, S. van der Meer, F. Farley, F. Krienen et al. The signal was observed for $\sim 130$ microseconds. A disagreement of 1.7 sigmabetween experiment and theory led to a correction of the latter.

A better experiment started in 1969 , using the so-called magic $\gamma$-factor at $3.1 \mathrm{GeV}$, the energy at which the electric field does not affect the g-2 precession, so that one can use electric quadrupoles with a uniform magnetic field. The signal was observed for 534 microsecond, the agreement with theory was excellent.

In his tribute to E. Picasso, F. Farley [3] underlined the excellent atmosphere in the group: "no wars, no clashes".

Figures 15 to 17 illustrate various aspects of the $\mathrm{g}-2$ program.

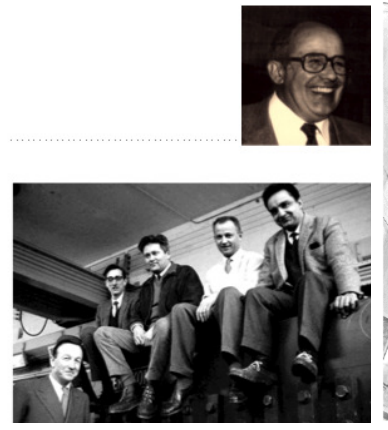

a

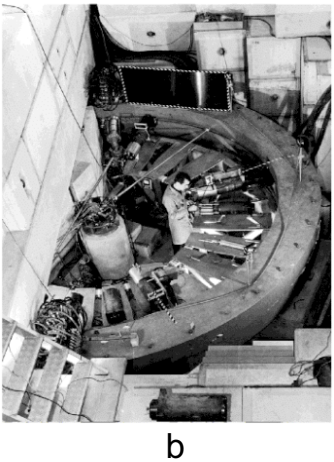

b

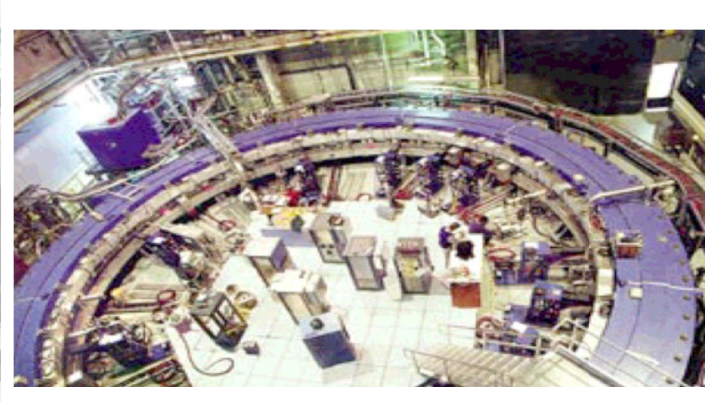

C

Figure 15 a: E.Picasso and the team of the first experiment; b: the first ring; c: the Brookhaven ring, now in Fermilab.

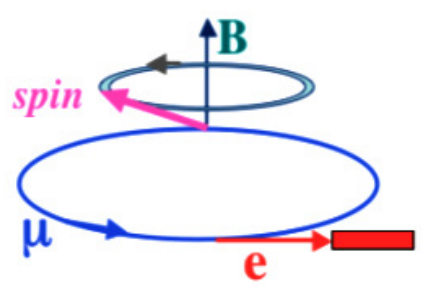

a

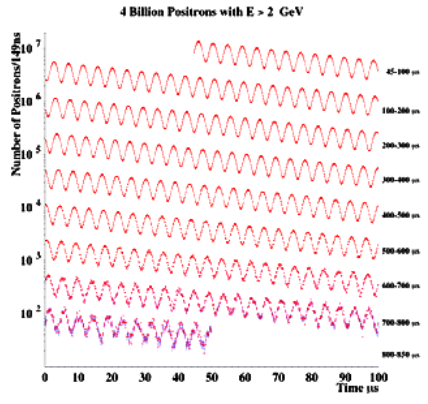

b

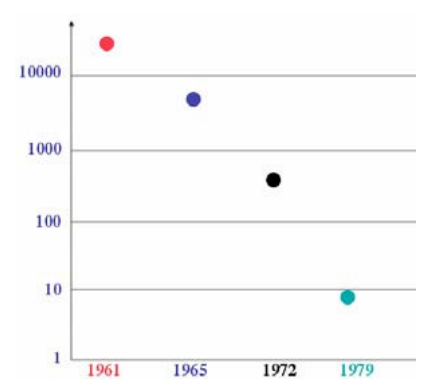

C

Figure 16. a: principle of the set up; b: the Brookhaven signal; c: decrease of CERN uncertainty. 

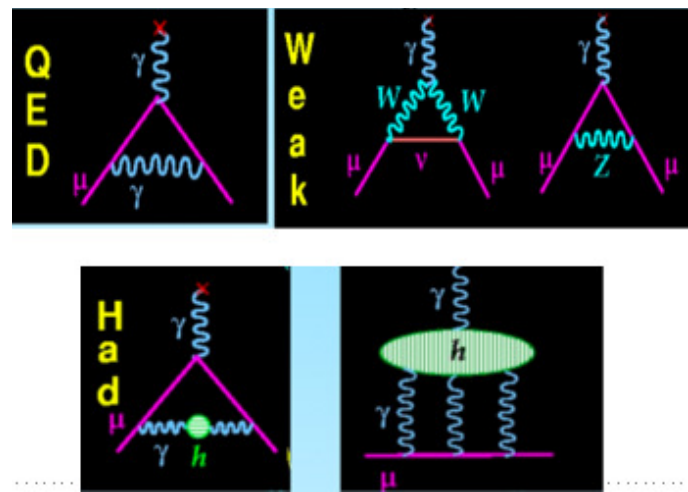

a

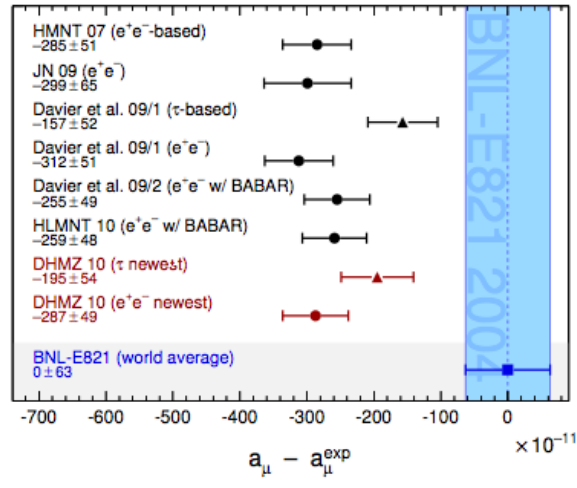

$\mathrm{b}$

Figure 17. a: the contributing diagrams; b: the present situation of theory versus experiment.

\section{LEP and ADLO}

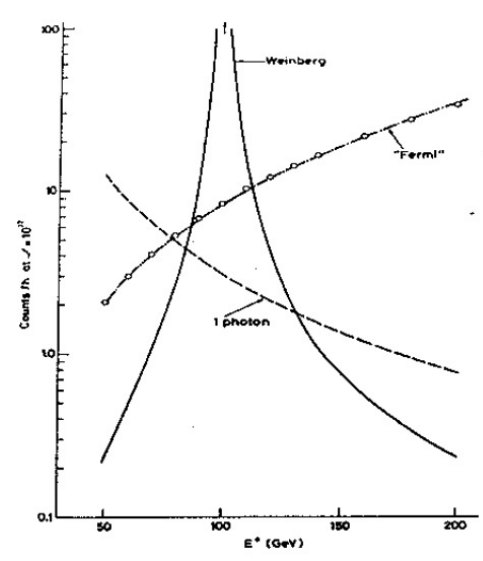

a

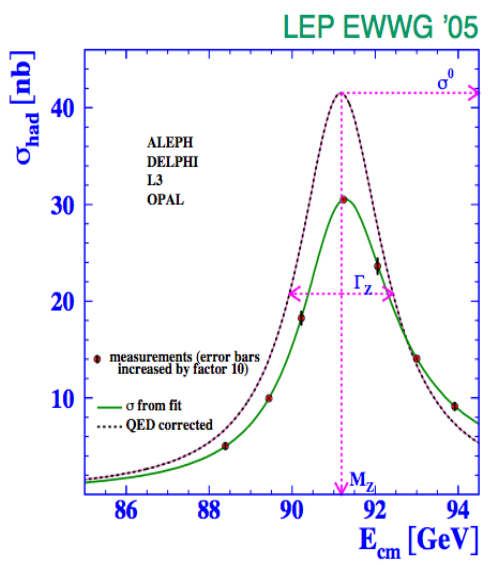

b

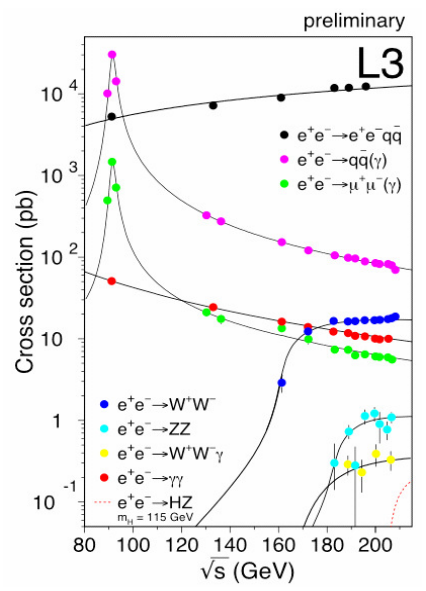

C

Figure 18. The LEP scenery; a: as anticipated by B.Richter in 1976; b and c: as observed.

ADLO is the acronym for the four experiments, ALEPH, DELPHI, L3 and OPAL, which combined their results, with a total of $17 \mathrm{M}$ hadronic $\mathrm{Z}^{0}$.

LEP is a well celebrated success story. The machine, planned very accurately 15 years in advance, worked magnificently, surpassing all expectations (see Table 2). The superconducting radiofrequency cavities (fig19 b,c), thanks to a careful work of conditioning and balancing, reached an accelerating field substantially higher than expected. The vacuum, with the large-scale use of getter pumping (fig 19a, C.Benvenuti), was outstanding, and this contributed to offer very favorable experimental conditions.

LEP was exploited by four high-quality multipurpose detectors. The program led to clean and subtle physics results: in particular it demonstrated the validity of the SM at the loop level. Through their effect as virtual objects, it gave results on particles too heavy to be 'really' produced, the top quark (fig 24 a) and the Higgs boson (fig 29 a). It showed that the coupling 'constants' of the three forces, electromagnetic, weak and strong, are actually running and converge exactly at a very high scale $\left(\sim 10^{16} \mathrm{GeV}\right)$ in the supersymmetric version of the SM. We briefly quote below the main LEP messages. 
We recall that, besides the main program, two additional variants of LEP were studied. Even if they were not realized, these studies had interesting consequences. The first was an attempt to exploit the longitudinal polarization of $\mathrm{e}^{ \pm}$at LEP. It turned out to be complicated and, since it would have delayed the LEP energy upgrade, it was not implemented. It led to the invention of a clever scheme, called the Blondel scheme, which may play an important role in the future at a $\mathrm{Z}$ factory, and to exploit transverse polarization, key to the precise measurement of beam energy and of the $\mathrm{Z}$ mass (figure 20c)

The second variant aimed at a much increased luminosity by devising multibunch schemes (Pretzel trains later replacing equidistant bunches in the Pretzel scheme). Actually up to 16 bunches were used, and LEP can be considered as a first approximation of a $\mathrm{Z}$ and therefore of a heavy-flavour factory.

Table 2. Performances, foreseen and achieved, for the machine and some physics observables.

LEP: design and reality.

\begin{tabular}{lll} 
Parametcr & $\begin{array}{l}\text { Design } \\
(55 / 95 \mathrm{GcV})\end{array}$ & $\begin{array}{l}\text { Achieved } \\
(46 / 98 \mathrm{GcV})\end{array}$ \\
\hline Bunch Current & $0.75 \mathrm{~mA}$ & $1.00 \mathrm{~mA}$ \\
Total Bcam Current & $6.0 \mathrm{~mA}$ & $8.4 \mathrm{~mA} / 6.2 \mathrm{~mA}$ \\
Vcrtical Bcam-beam parameter & 0.03 & $0.045 / 0.083$ \\
Emittance ratio & $4.0 \%$ & $0.4 \%$ \\
Maximum Luminosity & $16 / 2710^{30} \mathrm{~cm}^{-2} \mathrm{~s}^{-1}$ & $34 / 10010^{30} \mathrm{~cm}^{-2} \mathrm{~s}^{-1}$ \\
Horizontal beta function at IP & $1.75 \mathrm{~m}$. & $1.25 \mathrm{~m}$. \\
Vertical beta function at IP & $7.0 \mathrm{~cm}$. & $4.0 \mathrm{~cm}$. \\
\hline
\end{tabular}

In all respects $L E P$ did better, sometimes much better, than expected

\begin{tabular}{|c|c|c|}
\hline \multicolumn{2}{|r|}{ anticipated } & final \\
\hline $\mathbf{M}_{\mathbf{Z}}(\mathrm{MeV})$ & $\pm 10-15$ (stat) \pm 17 syst & \pm 2.1 \\
\hline$\Gamma_{\mathrm{Z}}(\mathrm{MeV})$ & $\pm \mathbf{5 0}$ & \pm 2.3 \\
\hline Normalisa & tion $\quad \mathbf{3 \%}$ & $<1 \%$ \\
\hline $\mathbf{M}_{\mathrm{W}}(\mathrm{MeV})$ & $50-60$ (stat) $>100$ (syst) & ADLO: 42 \\
\hline $\mathbf{A}_{\mathrm{FB}}^{\mu \mu}$ & twice better & \\
\hline$\tau$ polar. & 2.5 times better & \\
\hline $\mathbf{R}_{\mathrm{b}}$ & 3 to 6 times better & \\
\hline $\mathbf{A}_{\mathrm{FB}}{ }^{b}$ & 3 times better & \\
\hline
\end{tabular}

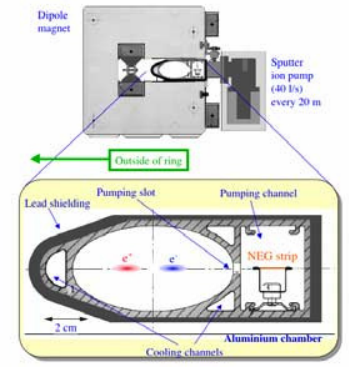

a

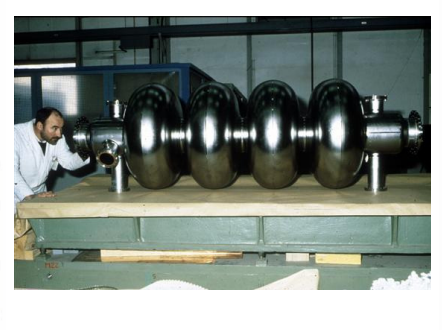

b

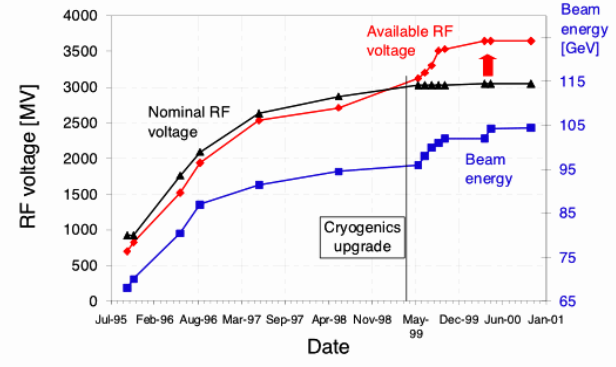

C

Figure 19. a: the LEP vacuum chamber; b: a SC RF cavity; c: evolution of the LEP RF voltage.

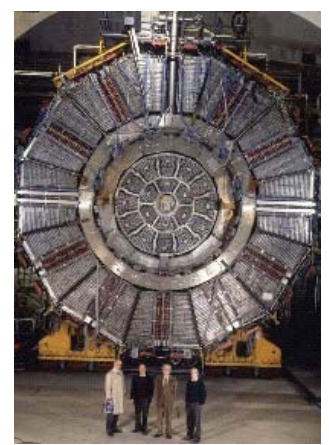

a

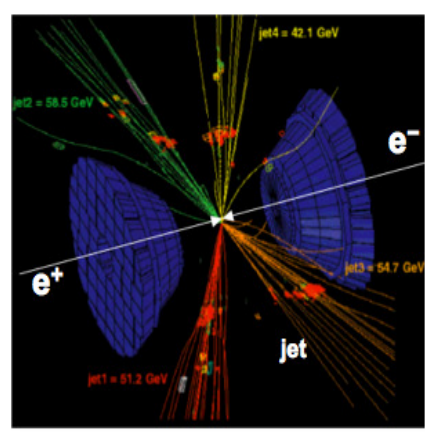

b

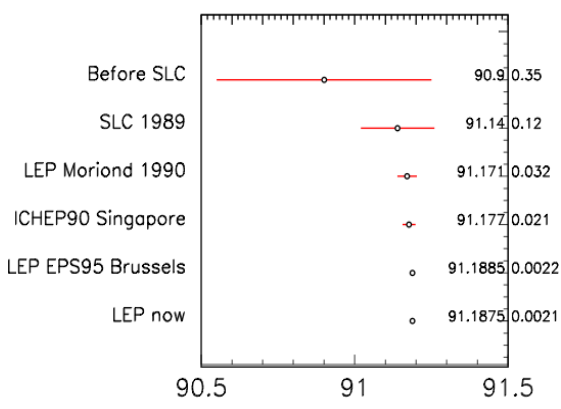

C

Figure 20. a: the ALEPH detector; b: a four-jet event in Delphi; c: the $Z^{0}$ mass measurement. 
On the experimental side one can underline:

- the use of high-performance luminometers and the most accurate theoretical knowledge of the relevant Bhabha cross-section (fig 21a), a key ingredient of neutrino counting (fig 22);

- the exploitation for beauty tagging of high-quality silicon microvertex detectors (fig $21 \mathrm{c}, \mathrm{d}$ ), allowing excellent purity for a still reasonable efficiency (fig $21 \mathrm{~b}$ ).

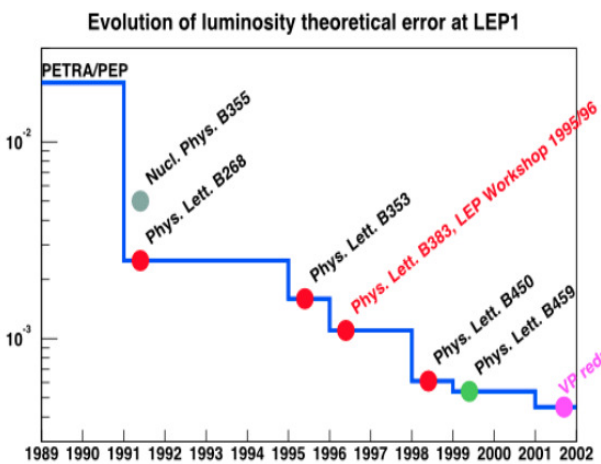

a

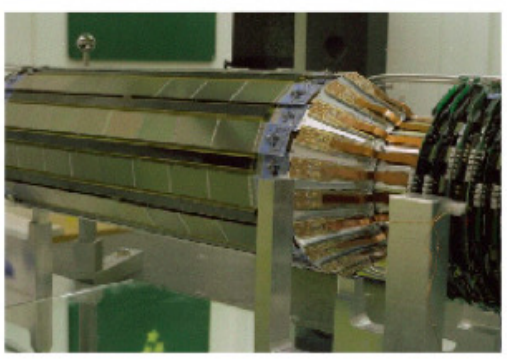

C

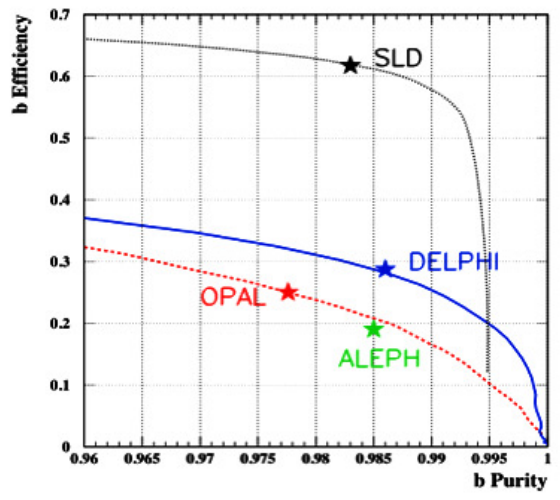

b

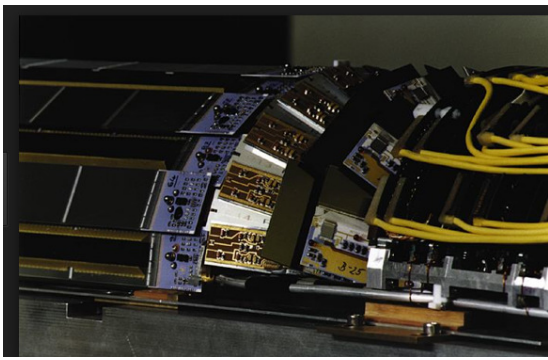

d

Figure 21. a: progress of the theoretical accuracy of the Bhabha cross-section; b: beauty tagging at LEP, performances and comparison with SLD CCD; c,d: Delphi microvertex and pixel detectors.

\subsection{LEP accurate measurements}

Figures 22 to 26 illustrate well-known results from LEP. Comments will be given in the legends.

An important but trivial quantum effect is the running of the fine structure constant from low energy to the $\mathrm{Z}$ mass, $\Delta \alpha$. In addition, did we feel other effects due in particular to the top and Higgs? The answer is a clear yes. The effects are summarized by the $\Delta \rho$ and $\Delta \mathrm{r}$. factors, whose numerical values give indirect information on these particles, as shown below (ref 4).

From the $\mathrm{Z}$ branching ratio into beauty, $\mathrm{R}_{\mathrm{b}}$ : the predicted value, neglecting radiative corrections, is $\mathrm{R}_{\mathrm{b}}{ }^{0}=0.2183$, the measured one is $\mathrm{R}_{\exp }=0.21644 \pm 0.00065$. From the $2.8 \sigma$ discrepancy one derives: $\mathrm{M}_{\mathrm{t}}=155 \pm 20 \mathrm{GeV}$.

- From the $\mathrm{Z}$ leptonic width: one finds $\mathrm{g}_{\mathrm{A}}{ }^{1}=-0.50123 \pm 0.00026,4.7$ sigmaaway from the canonical value of $-1 / 2$. One deduces $\Delta \rho=0.005 \pm 0.001$.

- From the W mass and its relation with $\mathrm{G}_{\mu}$ : one finds $\Delta \mathrm{r}=0.033 \pm 0.002$ or $\Delta \mathrm{r}_{\mathrm{w}}=\Delta \mathrm{r}-\Delta \alpha=$ $=-0.026 \pm 0.002,13 \sigma$ away from 0 ! 


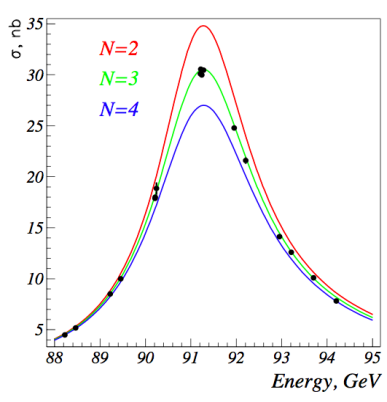

a

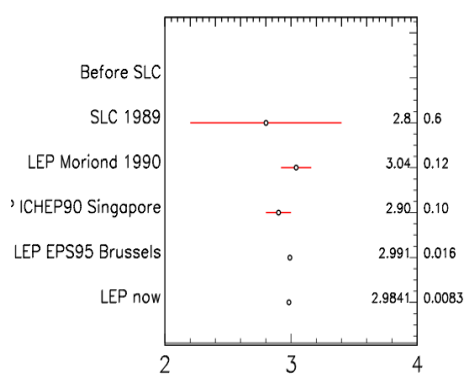

b

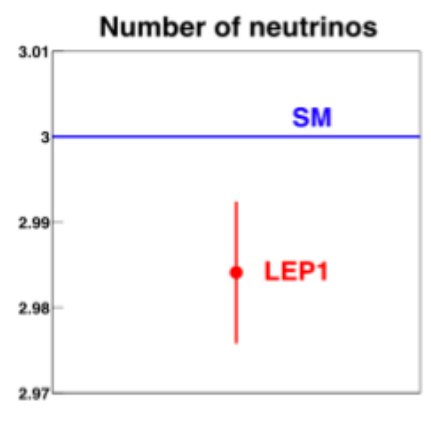

C

Figure 22. a: $Z$ line shape; b: the number of neutrinos; c: zoom on $N_{v}$ (about $2 \sigma$ below 3 ).

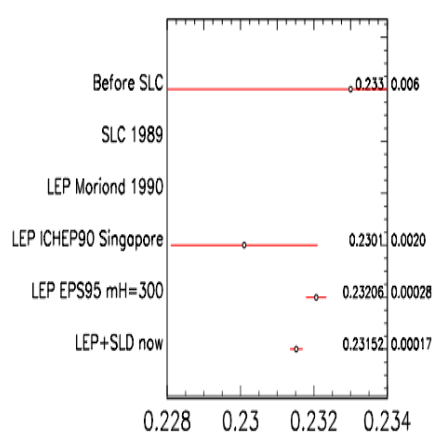

a

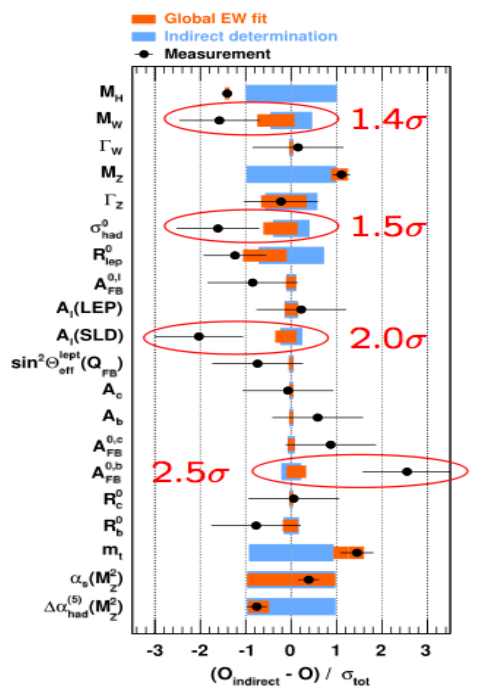

b
W-Boson Mass [GeV]

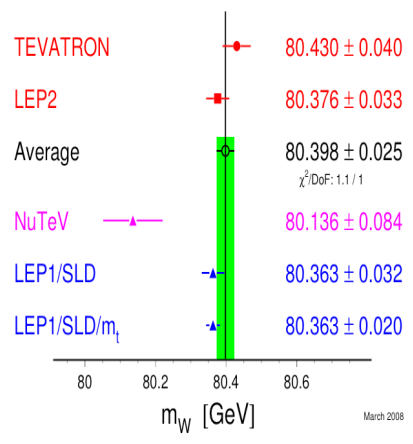

C

Figure 23. a: the weak angle $\sin ^{2} \theta_{\text {eff }}$; b: the global electroweak fit; c: the W mass situation in 2008

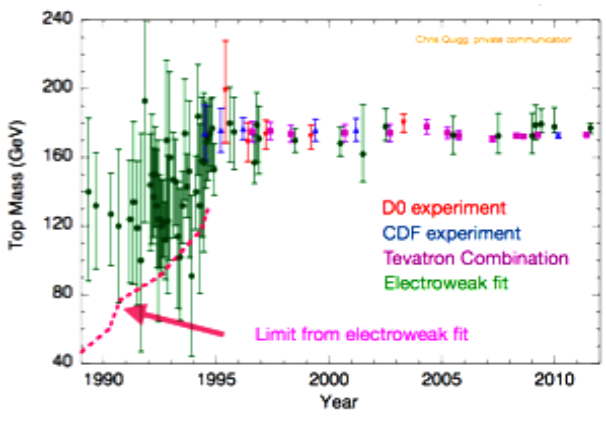

a

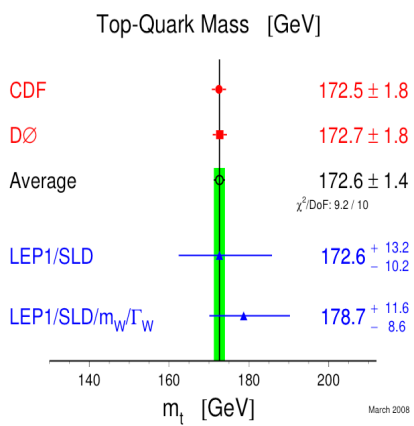

b

Figure 24. a: LEP prediction of the $\mathrm{M}_{\mathrm{top}}$ (green) and direct measurements of the Tevatron 


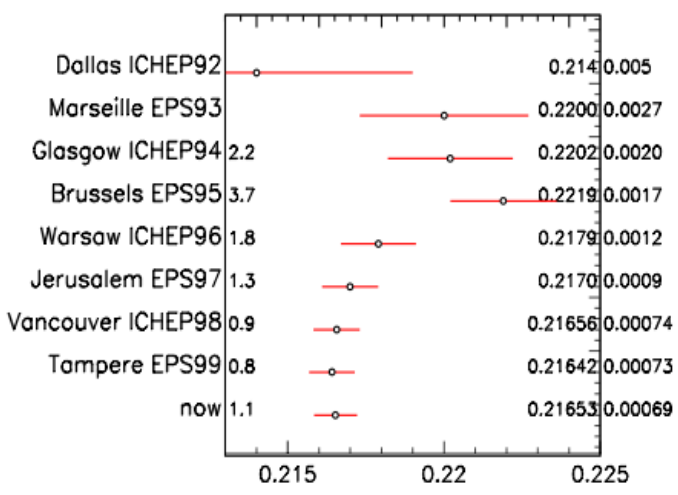

a

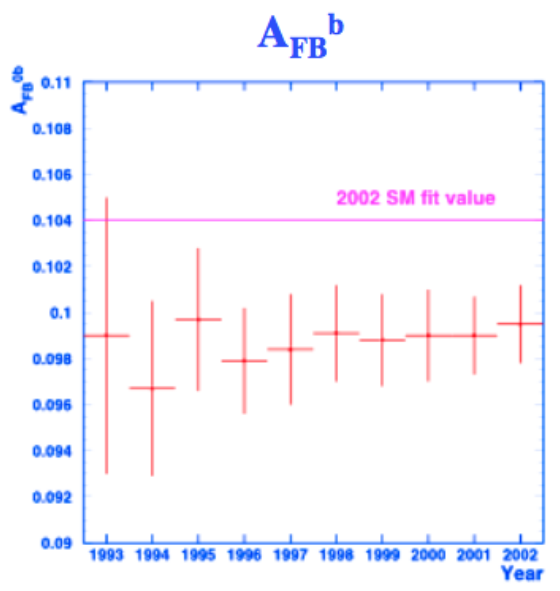

b

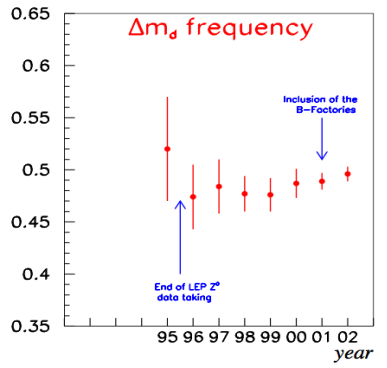

C

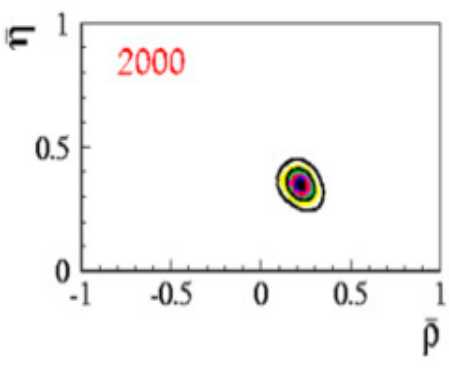

d

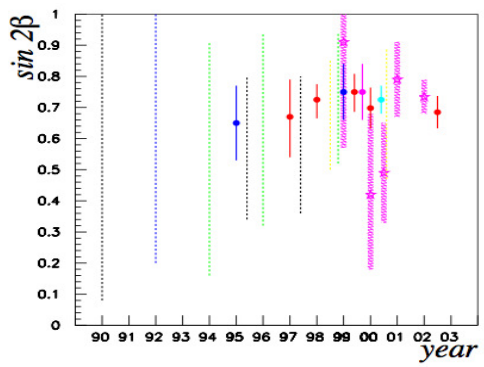

e

Figure 25. Beauty physics at LEP. a: evolution with time of $\mathrm{R}_{b}$; b: a discrepancy which stayed, $\mathrm{A}_{\mathrm{FB}}{ }^{\mathrm{b}}$; $\mathrm{c}$ : the $\mathrm{B}^{0}$ - antiB ${ }^{0}$ oscillation frequency; d: evidence for $\mathrm{CP}$ violation in the $\mathrm{B}$ sector; e: determination of the angle ${ }^{\circ}$, indirect and direct (pink, at B factories).

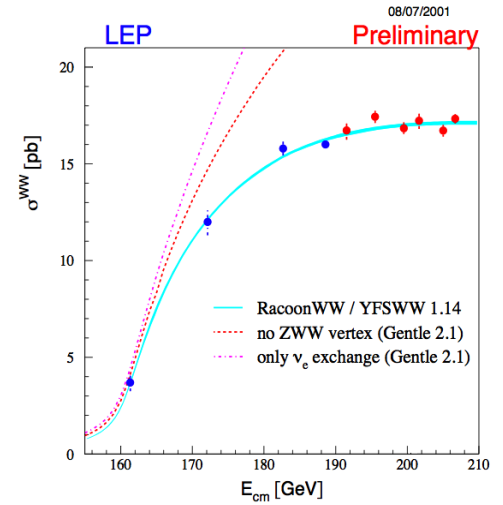

a

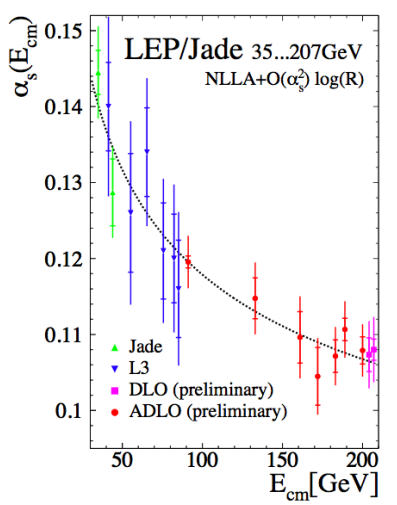

b

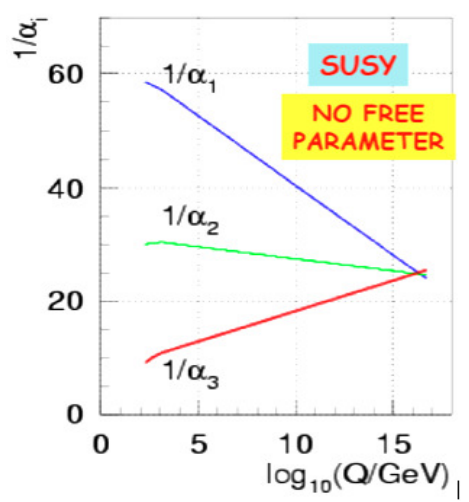

C

Figure 26. a: proof of the non abelian nature of the electroweak theory through the $\mathrm{W}$ pair cross-section; b: the running of the strong coupling with the energy scale; c: the running of the coupling constants of the three forces in Supersymmetry. 
Running is due to the role, as virtual objects, of the particles existing in the model considered. A supersymmetric version of the SM leads to a perfect convergence at about $10^{16} \mathrm{GeV}$ provided some superpartners are relatively light.

\subsection{The BEH boson at LEP}

Before LEP, there was basically no limit on the BEH boson mass. The 1988 PDG said that, probably, it was heavier than $4 \mathrm{GeV}$. LEP1 (on the $\mathrm{Z}^{0}$ ) brought the limit to $63 \mathrm{GeV}$.

Figures 27 to 34 illustrate the search for the SM-like Higgs boson at LEP2 (above the $Z^{0}$ ). The production mode, a tree level process, with a threshold at $\mathrm{M}_{\mathrm{Z}}+\mathrm{M}_{\mathrm{h}}$, the cross-section and backgrounds are shown in figure $28 \mathrm{a}, \mathrm{b}$.

In brief, the maximum useful $\mathrm{CM}$ energy reached was $206 \mathrm{GeV}$ in 2000 . A lower limit on the boson mass was set at $114 \mathrm{GeV}$. Since then, LHC told that a weak signal at $115 \mathrm{GeV}$ (figure $28 \mathrm{c}$ ) was fake and found the boson at $125 \mathrm{GeV}$.

In 2000 LEP2 had 285 superconducting RF cavities. More cavities had been asked since 1994 to get an answer about the light boson $(<130 \mathrm{GeV})$ predicted by minimal SUSY. Hundred more could have been installed in the equipped zones of LEP and would have raised the CM energy to $220 \mathrm{GeV}$. But the production of cavities was stopped in 1996.

SUSY or not, a light boson was there and LEP2 missed it. Figure 29 shows the final legacy of LEP.

More information in the legends of the figures.
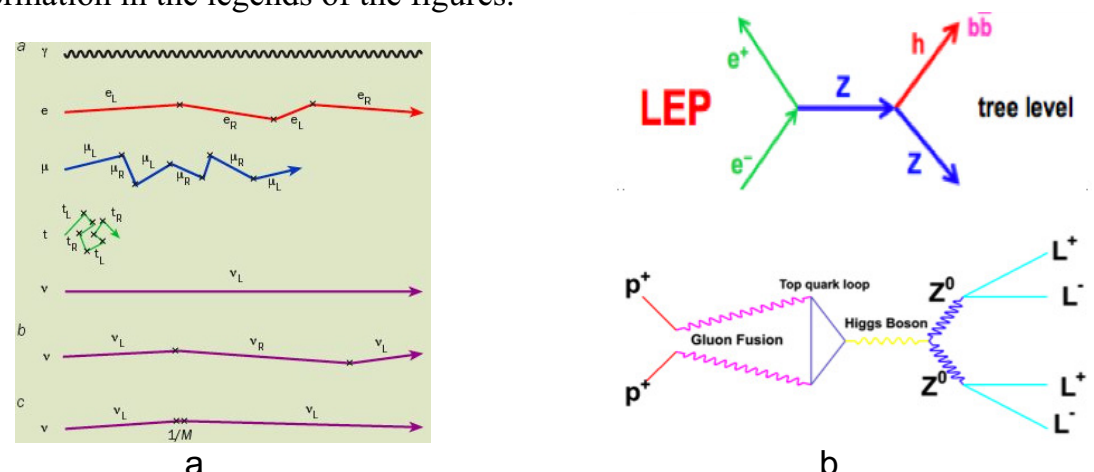

b

Figure 27. a: Murayama's view of how particles get their mass in the BEH field; b: the boson production modes at LEP2 (tree level, provided $2 \mathrm{E}_{\mathrm{b}}>\mathrm{M}_{\mathrm{Z}}+\mathrm{M}_{\mathrm{h}}$ ) and LHC (loops involved).

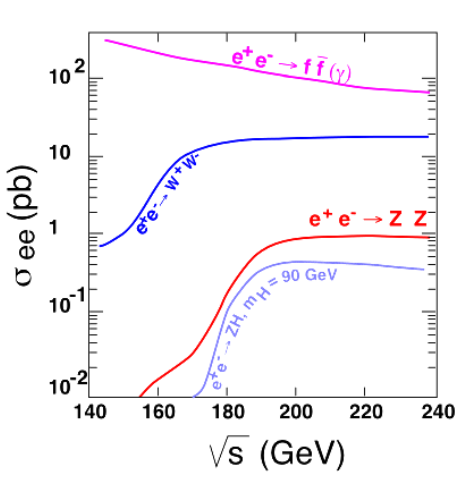

a

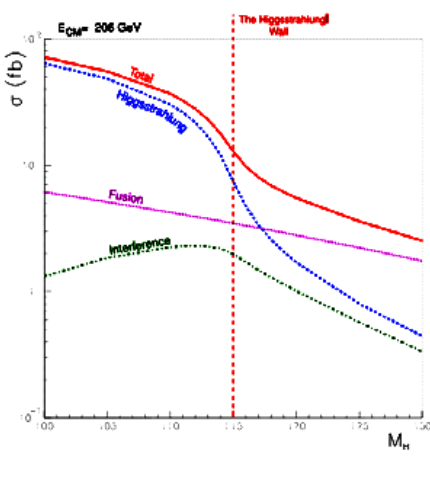

b

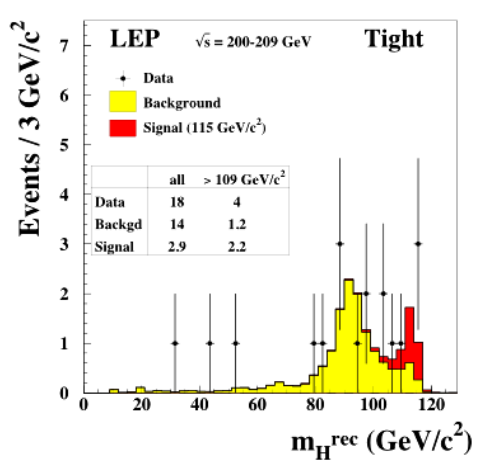

C

Figure 28. Higgs search at LEP200: a,b: cross-sections c: the final mass spectrum of ADLO. In 2000 LEP206 hit the kinematical limit for $\mathrm{M}_{\mathrm{h}}=115 \mathrm{GeV}$. It was impossible to increase more the energy. 


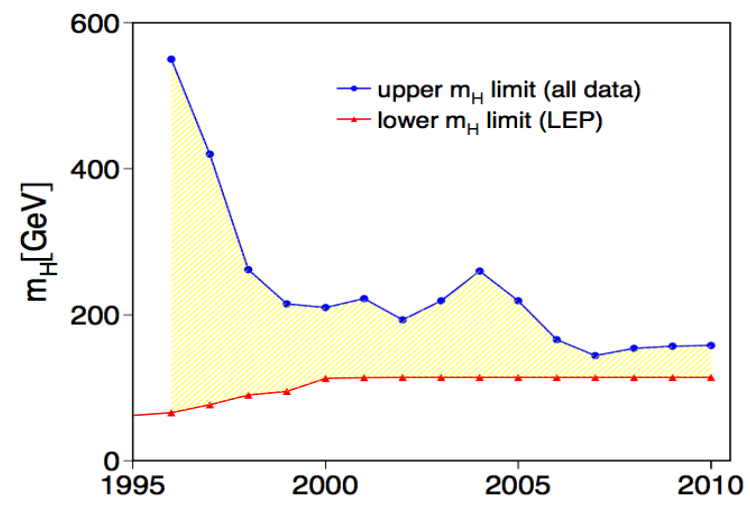

a

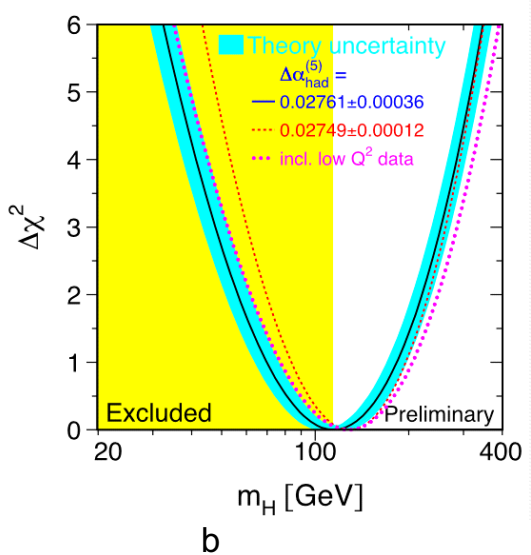

b

Figure 29. a: LEP information on the mass of the boson. In red the lower limit from the search, in blue the upper limit from the electroweak fit; b: the usual chi2 plot in the SM.

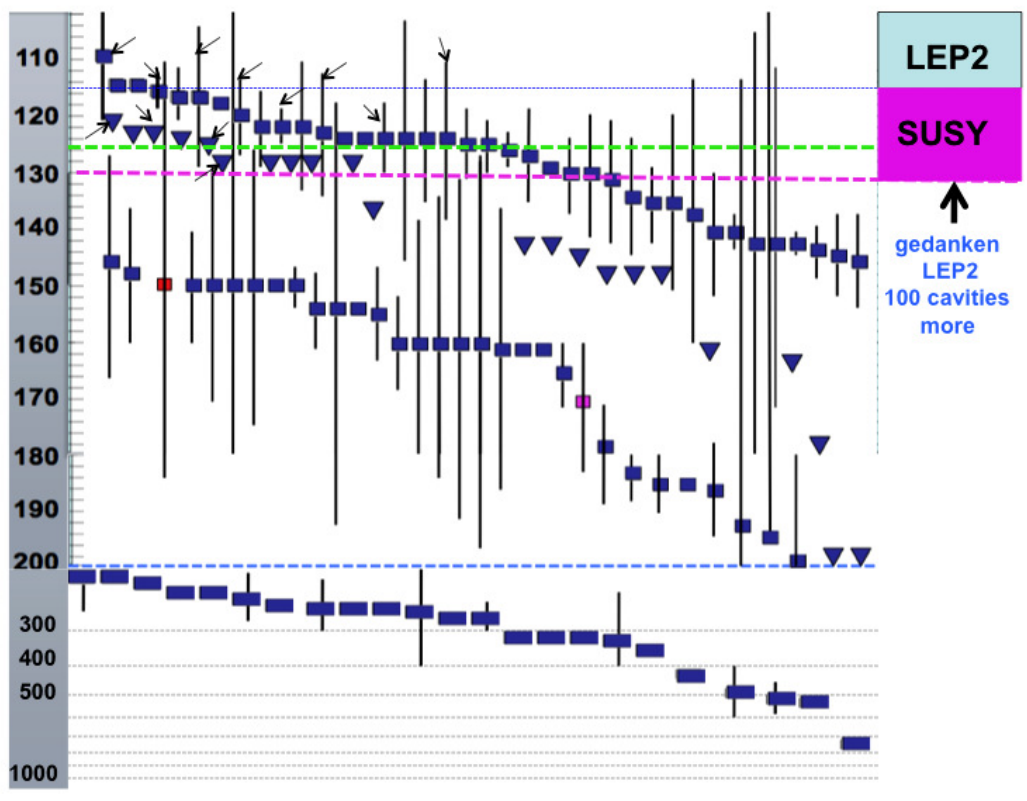

Figure 30. Compilation of the theoretical "predictions" of the BEH boson mass (from [5]). Arrows indicate those referring to SUSY models. A triangle gives an upper limit. The potential impact that a modest energy rise of LEP200 with 100 more superconducting RF cavities is shown. 


\section{Apologies and conclusion}

It is clear that several other important topics and contributions of CERN should be mentioned and developped.

For instance the BC saga at CERN and the SPS program, quoted in part 2, deserve much more.

Similarly the vast amount of information, from all programs, which helped building and validating QCD needs to be thoroughly described, in particular the heavy ion program which led CERN in 2000 to announce the creation of "a new state of matter".

This is also true for the intense activity concerning discrete symmetries, following the discovery in 1964 of CP violation (SPS, NA31 and NA48, LEP, LEAR, AD), presently a major topic at LHC, following the $\mathrm{B}$ factories.

Finally the field of rare decays, possible window on new physics, is a most interesting one, well covered at CERN, for instance by NA62.

I refer to [1] for a more complete account of these subjects in the first 50 years of CERN life and to several historical talks given during this year of the 60 anniversary of CERN [7].

One can also notice many illuminating papers, explaining, as simply as possible, some physics topics. Those of [6] are most stimulating examples.

In summary, and coming back to Table 1 , I think we have been living amazing decades, bringing particle physics from a kind of "entomology" in the sixties to the coherent picture and subtlety of the particle SM. Cosmology also has now its "concordance model". This mutation is due to bright theoretical ideas, to bright technical and instrumental developments, in our field and outside, to the enthusiasm and devotion of many actors.

One should also be grateful to the public support which allowed us to build the giant machines needed. Conversely the success of bigger and bigger worldwide collaborations is a most remarkable achievement of our community and a good example offered to the outside world.

However it is also obvious that the more we learn, the more we bump on new questions. The LHC and various programs at the high intensity frontier, in non-accelerator physics and in astrophysics offer the chance to understand more. There is no guarantee of success, but we must exploit them at best and without too many preconceived ideas.

Without hiding shadows, CERN, well thought from the start, is a great success, with a most impressive bilan of discoveries and accurate measurements: NC, W and Z, LEP physics, BEH boson. Let's hope this will go on and CERN will keep its leadership at the HE frontier.

\section{Acknowledgements}

I would like to thank L.Pape, R.Barate and F. Pauss for their careful reading of the manuscript and their comments.

\section{References}

1. For the material shown in the 2004 Academic training on Fifty Years of research at CERN, from past to future: http://indico.cern.ch/event/a043644/. For the written version of the 50 years Academic Training http://delphiwww.cern.ch/talks/general/Treille.50ans/50ans treille.pdf; see also:

Report CERN-2005-008 (in French); Highlights of 25 years of physics at CERN, Phys. Rep. 62 (1980);

CERN -The second 25 years, Phys. Rep. 403-404 (2004);

Reports CERN CHS-21 and CHS-23, U. Mersits (1987);

CERN CHS-26, L. Weiss (1988);

CERN CHS-36, D. Pestre (1992);

L. Di Lella, Phys. Rep. 225 (1993) 45;

D. Denegri, DAPNIA-04-247 (2004);

Report CERN CHS-20, J. Krige (1987). 
2. D. Haidt, CERN Courier 44 No. 8 (2004) 21-24; Eur. Phys. J. C34 (2004) 25-31;

A. Rousset, Nucl. Phys. Proc. Suppl. 36 (1994) 339-361;

D. Perkins, CERN Courier 43 No. 5 (2003) Suppl. 15-19; http://cerncourier.com/main/article/43/5/22 .

3. F. Farley, G minus 2 plus Emilio, CERN-OPEN-2002-006 (1992).

4. F. Teubert, Int. J. Mod. Phys. A20 (2005) 5174-83; LEP EW Group, http://lepewwg.web.cern.ch/LEPEWWG/ .

5. Higgs mass predictions, Thomas Schucker, $0708.3344 \mathrm{v} 8$

6. F. Wilczek, Mass without mass, Phys. Today 53 No.1 (2000) 13-14. F. Wilczek, QCD and natural philosophy, MIT.CTP.3328 (2002), Archive: physics/0212025; P. Sikivie, Phys. Today 49 No.12 (1996) 22-27.

7. http://cern60.web.cern.ch/en/events, in particular talks by L. di Lella, L.Fayard. 